\title{
THE CHANDRA COSMOS SURVEY. I. OVERVIEW AND POINT SOURCE CATALOG
}

\author{
Martin Elvis $^{1}$, Francesca Civano $^{1}$, Cristian Vignali $^{2,3}$, Simonetta Puccetti $^{4,5}$, Fabrizio Fiore $^{5}$, Nico Cappelluti ${ }^{6}$, \\ T. L. Aldcroft ${ }^{1}$, Antonella Fruscione ${ }^{1}$, G. $_{\text {Zamorani }}{ }^{3}$, Andrea Comastri $^{3}$, Marcella Brusa ${ }^{6,7}$, Roberto Gilli $^{3}$, \\ Takamitsu Miyaji ${ }^{8,9,30}$, Francesco Damiani ${ }^{10}$, Anton M. Koekemoer ${ }^{11}$, Alexis Finoguenov ${ }^{6,7}$, Hermann Brunner ${ }^{6}$, \\ C.M. Urry ${ }^{12}$, John Silverman $^{13}$, Vincenzo Mainieri $^{14}$, Guenther Hasinger $^{6,15}$, Richard GrifFiths $^{16}$,

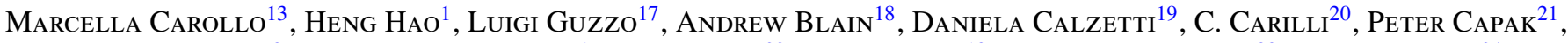 \\ Stefano EtTori $^{3}$, Giuseppina Fabbiano ${ }^{1}$, Chris Impey ${ }^{22}$, Simon Lilly $^{13}$, Bahram Mobasher ${ }^{23}$, Michael Rich $^{24}$, \\ Mara Salvato ${ }^{18}$, D.B. Sanders ${ }^{25}$, Eva Schinnerer ${ }^{26}$, N. Scoville ${ }^{18}$, Patrick Shopbell ${ }^{18}$, James E. Taylor $^{27}$, \\ YOSHIAKI TANIGUCHI ${ }^{28}$, AND MARTA VOLONTERI ${ }^{29}$ \\ ${ }^{1}$ Harvard-Smithsonian Center for Astrophysics, 60 Garden St., Cambridge, MA 02138, USA \\ 2 Dipartimento di Astronomia, Università degli Studi di Bologna, Via Ranzani 1, I-40127 Bologna, Italy \\ ${ }^{3}$ INAF-Osservatorio Astronomico di Bologna, Via Ranzani 1, I-40127 Bologna, Italy \\ ${ }^{4}$ ASI Science Data Center, via Galileo Galilei, 00044 Frascati, Italy \\ ${ }_{5}^{5}$ INAF-Osservatorio astronomico di Roma, Via Frascati 33, 00040 Monteporzio Catone, Italy \\ ${ }^{6}$ Max-Planck-Institute für Extraterrestrische Physik, Postfach 1312, 85741, Garching bei München, Germany \\ ${ }^{7}$ University of Maryland, Baltimore County, 1000 Hilltop Circle, Baltimore, MD 21250, USA \\ ${ }^{8}$ Instituto de Astronomía, Universidad Nacional Autónoma de México, Ensenada, Mexico \\ ${ }^{9}$ Center for Astrophysics and Space Sciences, University of California San Diego, Code 0424, 9500 Gilman Drive, La Jolla, CA 92093, USA \\ ${ }^{10}$ INAF-Osservatorio Astronomico di Palermo, Piazza del Parlamento 1, I-90134 Palermo, Italy \\ ${ }^{11}$ Space Telescope Science Institute, 3700 San Martin Drive, Baltimore, MD 21218, USA \\ ${ }^{12}$ Department of Physics and Yale Center for Astronomy \& Astrophysics, Yale University, P.O. Box 208121, New Haven, CT 06520-8121, USA \\ ${ }_{13}$ Department of Physics, Eidgenössische Technische Hochschule-Zurich, CH-8093 Zurich, Switzerland \\ ${ }^{14}$ ESO, Karl-Schwarschild-Strasse 2, D-85748 Garching, Germany \\ ${ }^{15}$ Max-Planck-Institute für Plasmaphysik, Boltzmannstrasse 2, D-85748 Garching bei München, Germany \\ ${ }^{16}$ Department of Physics, Carnegie Mellon University, 5000 Forbes Avenue, Pittsburgh, PA 15213, USA \\ ${ }^{17}$ INAF-Osservatorio Astronomico di Brera, via Bianchi 46, 23807 Merate, Italy \\ ${ }^{18}$ California Institute of Technology, MC 105-24, 1200 East California Boulevard, Pasadena, CA 91125, USA \\ ${ }^{19}$ Department of Astronomy, University of Massachusetts, Amherst, MA 01003, USA \\ ${ }^{20}$ National Radio Astronomy Observatory, PO Box O, Socorro NM 87801, USA \\ ${ }^{21}$ Spitzer Science Center, 314-6 Caltech, Pasadena, CA 91125, USA \\ ${ }^{22}$ Steward Observatory, University of Arizona, 933 North Cherry Avenue, Tucson, AZ 85721, USA \\ ${ }^{23}$ Department of Physics and Astronomy, University of California, Riverside, CA 92521, USA \\ ${ }^{24}$ Department of Physics and Astronomy, University of California, Los Angeles, CA 90095, USA \\ ${ }^{25}$ Institute for Astronomy, University of Hawaii, 2680 Woodlawn Dr., Honolulu, HI 96822, USA \\ ${ }^{26}$ Max-Planck-Institut für Astronomie, Königstuhl 17, Heidelberg D-69117, Germany \\ 27 Department of Physics and Astronomy, University of Waterloo, Waterloo, Ontario, N2L 3G1, Canada \\ ${ }^{28}$ Research Center for Space and Cosmic Evolution, Ehime University, Bunkyo-cho 2-5, Matsuyama 790-8577, Japan \\ ${ }^{29}$ Department of Astronomy, University of Michigan, Ann Arbor, MI 48109, USA \\ Received 2008 November 4; accepted 2009 July 29; published 2009 August 27
}

\begin{abstract}
The Chandra COSMOS Survey (C-COSMOS) is a large, $1.8 \mathrm{Ms}$, Chandra program that has imaged the central $0.5 \mathrm{deg}^{2}$ of the COSMOS field (centered at $10^{h},+02^{\circ}$ ) with an effective exposure of $\sim 160 \mathrm{ks}$, and an outer $0.4 \mathrm{deg}^{2}$ area with an effective exposure of $\sim 80 \mathrm{ks}$. The limiting source detection depths are $1.9 \times 10^{-16} \mathrm{erg} \mathrm{cm}^{-2} \mathrm{~s}^{-1}$ in the soft $(0.5-2 \mathrm{keV})$ band, $7.3 \times 10^{-16} \mathrm{erg} \mathrm{cm}^{-2} \mathrm{~s}^{-1}$ in the hard (2-10 keV) band, and $5.7 \times 10^{-16} \mathrm{erg} \mathrm{cm}^{-2} \mathrm{~s}^{-1}$ in the full $(0.5-10 \mathrm{keV})$ band. Here we describe the strategy, design, and execution of the C-COSMOS survey, and present the catalog of 1761 point sources detected at a probability of being spurious of $<2 \times 10^{-5}$ (1655 in the full, 1340 in the soft, and 1017 in the hard bands). By using a grid of 36 heavily ( $\sim 50 \%)$ overlapping pointing positions with the ACIS-I imager, a remarkably uniform $( \pm 12 \%)$ exposure across the inner $0.5 \mathrm{deg}^{2}$ field was obtained, leading to a sharply defined lower flux limit. The widely different point-spread functions obtained in each exposure at each point in the field required a novel source detection method, because of the overlapping tiling strategy, which is described in a companion paper. This method produced reliable sources down to a 7-12 counts, as verified by the resulting $\log N-\log S$ curve, with subarcsecond positions, enabling optical and infrared identifications of virtually all sources, as reported in a second companion paper. The full catalog is described here in detail and is available online.
\end{abstract}

Key words: catalogs - cosmology: observations - galaxies: evolution - quasars: general - surveys - X-rays: general

Online-only material: color figures, supplementary data

\section{INTRODUCTION}

${ }^{30}$ Address for correspondence: P.O. Box 439027, San Ysidro, CA 92143-9027, USA.
The co-evolution of galaxies and quasars or active galactic nuclei (AGNs) has been vigorously pursued both observationally 

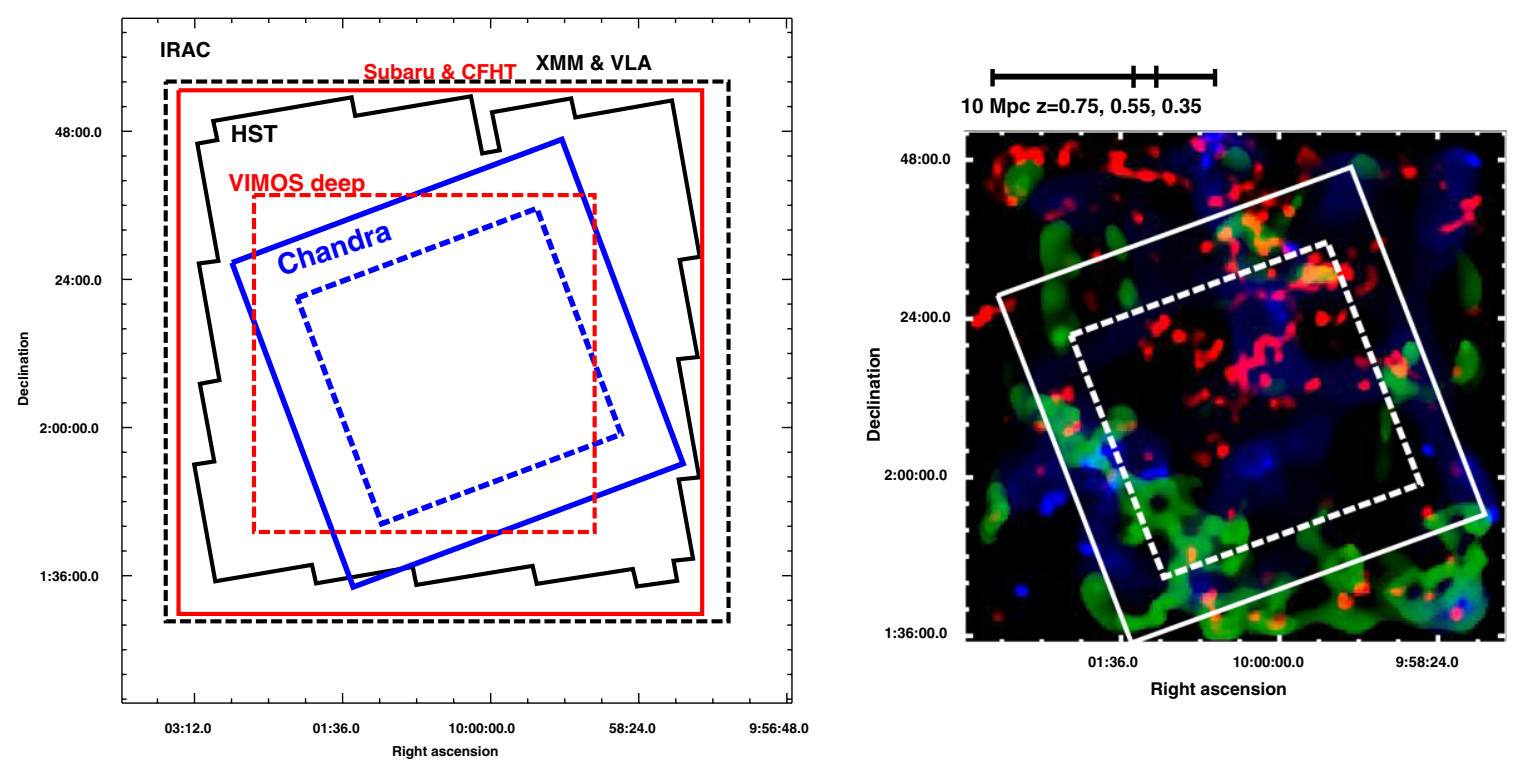

Figure 1. Left: map of the COSMOS field showing the coverage at various wavelengths: the IRAC $3.6 \mu \mathrm{m}$ mosaic is the background image; blue solid $=$ Chandra, blue dashed $=$ Chandra deep; black polygon $=H S T$; red solid = Subaru, CFHT, zCOSMOS bright; red dashed $=$ zCOSMOS deep; black dashed $=X M M$ and VLA The Spitzer MIPS observations cover an area two times larger. Right: LSS seen in galaxy distributions in the COSMOS field (Scoville et al. 2007c), ranging in size from 1-20 Mpc, based on photo-z's accurate to $\sim 1 \%$. Blue is centered on $z=0.35$, green on $z=0.55$, and red on $z=0.75$, each with $\Delta z=0.05$. The C-COSMOS field outline is shown as the white tilted square, with the dashed line delineating the high exposure area as in the left panel. A scale showing $10 \mathrm{Mpc}$ at the three redshifts is shown at the top. In both panels north is up, east is to the left.

and theoretically for a decade, ever since the discovery that the mass of the central black hole is tightly correlated both with the luminosity (Magorrian et al. 1998; Marconi \& Hunt 2003) and the velocity dispersion of the spheroid $\left(\mathrm{M}_{B H}-\sigma\right.$ relation; Ferrarese \& Merrit 2000; Gebhardt et al. 2000; Tremaine et al. 2002). Tackling this large subject requires the study of both galaxies and AGNs back to the epoch when both were growing rapidly, i.e. $z \sim 1-3$, requiring deep observations across many wavelengths, from radio through the infrared, optical and ultraviolet, to the X-rays. At the same time, the wide range of cosmic density and the rapid changes in this large-scale structure (LSS) require wide field observations that sample the universe at close to their true fractions.

The Cosmic Evolution Survey (COSMOS; Scoville et al. 2007a) is a deep and wide extragalactic survey designed to have sufficient area to overcome most cosmic variance, which requires sampling regions some $50 \mathrm{Mpc}$ on a side (Figure 1; Scoville et al. 2007a), and with sufficient depth to sample the $z=1-3$ galaxy and AGN population. The contiguous $2 \mathrm{deg}^{2}$ COSMOS field samples a volume of $\sim 6 \times 10^{6} \mathrm{Mpc}^{3}$ at $z=0.5-1$ (Wright 2006). This is $\sim 10 \%$ of the volume imaged by the Sloan Digital Sky Survey (SDSS) in the local $(z<0.1)$ universe $\left(5.7 \times 10^{7} \mathrm{Mpc}^{3}, 8000 \mathrm{deg}^{2}, \mathrm{DR}^{31}\right)$. COSMOS is a region of low, uniform, Galactic obscuration $\left(E(B-V) \simeq 0.02 \mathrm{mag}, \mathrm{N}_{H}\left(2.7 \times 10^{20} \mathrm{~cm}^{-2}\right.\right.$; Dickey \& Lockman 1990). COSMOS is likely to be the largest survey of this type for the next decade.

The location of the COSMOS area near the equator $\left(10^{\mathrm{h}}\right.$, $+02^{\circ}$ ) allows all major and future facilities ${ }^{32}$ (notably EVLA, ALMA, and SKA) to target this region down to faint limits (Scoville et al. 2007a). Space-based imaging has been undertaken in the F840W ( $\sim i$-band) with the Hubble Space Telescope (HST, Scoville et al. 2007b), in the $3.5 \mu \mathrm{m}-70 \mu \mathrm{m}$ infrared using Spitzer IRAC and MIPS (Sanders et al. 2007), in the

\footnotetext{
31 http://www.sdss.org/dr5/

32 Except for those in Antarctica.
}

UV using the Galaxy Evolution Explorer (GALEX; Zamojski et al. 2007), and in 0.5-10 keV X-rays with XMM (Hasinger et al. 2007; Cappelluti et al. 2007). Ground-based imaging spans the radio (1400 MHz VLA; Schinnerer et al. 2007), the near-IR with CTIO and KPNO (Capak et al. 2007) and CFHT (McCracken et al. 2009), the optical to AB 26-27 with Subaru in 21 bands (Taniguchi et al. 2007). Finally, large dedicated ground-based spectroscopy programs in the optical with Magellan/IMACS (Trump et al. 2007) and VLT/VIMOS (Lilly et al. 2007) are well under way.

This wealth of data has resulted in an initial 15-band photometric catalog of $\sim 10^{6}$ objects (Capak et al. 2007) from which photometric redshifts good to $<3 \%$ for $z<1.2$ and $r<24$ have been derived (Mobasher et al. 2007). Recently, more photometric bands have been added, resulting in improved photo- $z$ 's for the galaxy population accurate to $\Delta z /(1+z)<1 \%$ (Ilbert et al. 2008 ) and to $\Delta z /(1+z) \sim 2 \%$ for the AGN population (Salvato et al. 2009).

We have undertaken the Chandra-COSMOS survey (C-COSMOS) to cover the central $0.9 \mathrm{deg}^{2}$ region of the COSMOS field (Figure 1, left), containing a wide range of cosmic overdensity (Figure 1, right), with the ACIS-I CCD imager (Garmire et al. 2003) on board the Chandra X-ray Observatory (Weisskopf et al. 2002). The survey took 1.8 Ms of Chandra observing time ( $\sim 21$ days) and was the largest guest observer program approved in a single $\mathrm{AO}$ at the time it was undertaken (2006 November-2007 June). C-COSMOS employed a series of 36 heavily overlapped ACIS-I 50 ks pointings to give an exposure of $\sim 160 \mathrm{ks}$ over the inner area to a depth of $\sim 1.9 \times$ $10^{-16} \mathrm{erg} \mathrm{cm}^{-2} \mathrm{~s}^{-1}(0.5-2 \mathrm{keV})$, providing an unprecedented combination of contiguous area and depth in the X-ray band. This overlapping tiling strategy gives highly uniform exposure, and so a well-defined flux limit.

Several of the deepest COSMOS surveys are now concentrating on this same central subfield of COSMOS: the $z$-COSMOS Deep spectroscopic survey (to $B \sim 25$; Lilly et al. 2007), the deep VLA survey (6 $\mu \mathrm{Jy}$ rms; E. Schinnerer et al. 2009, in 


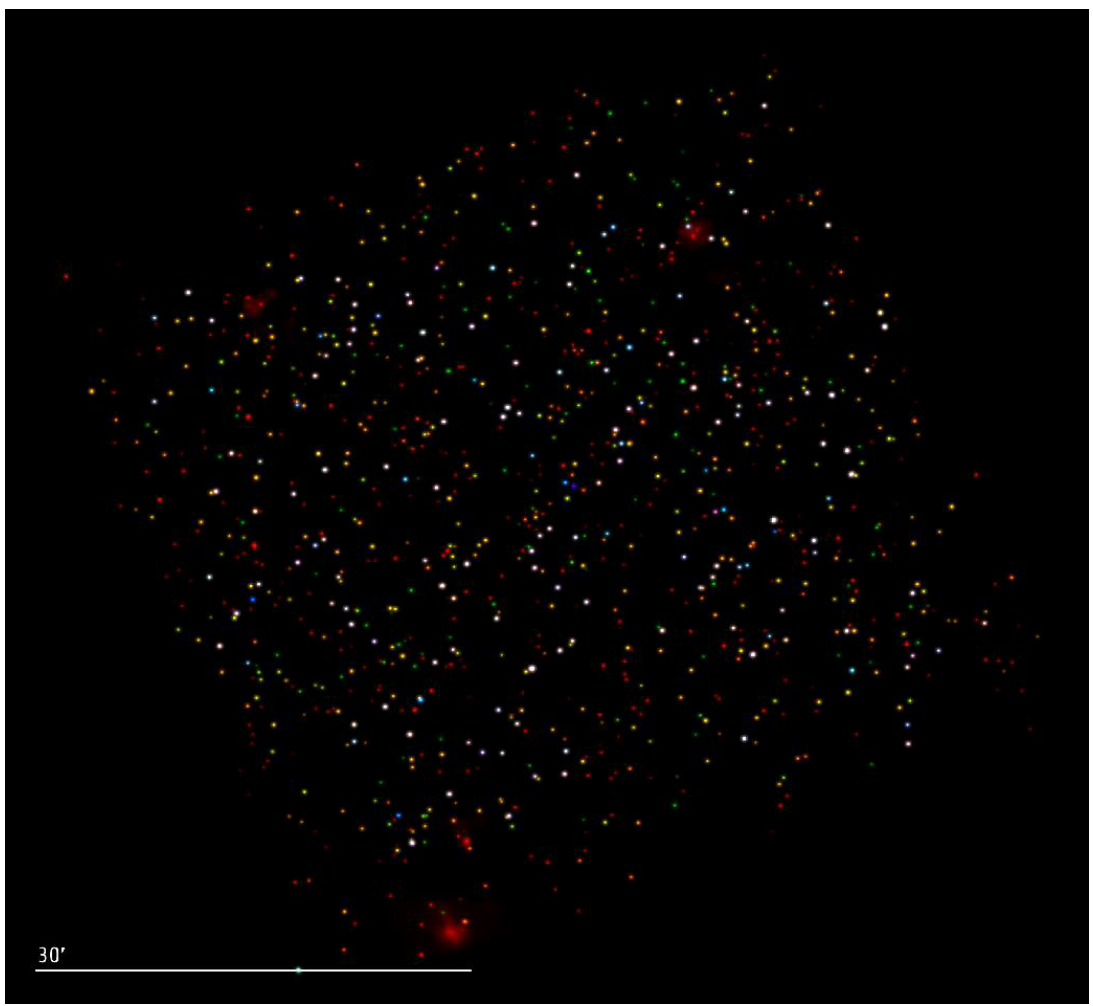

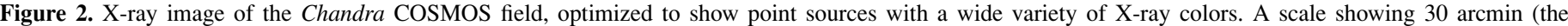

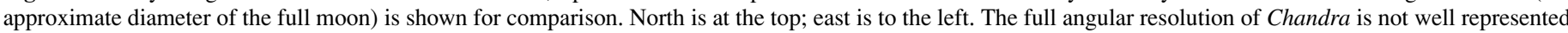

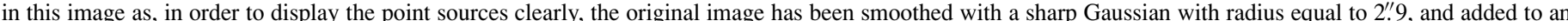

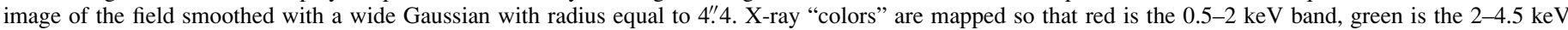

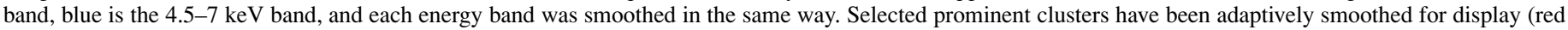
extended shapes).

preparation), and several millimeter and submillimeter surveys (MAMBO, Bertoldi et al. 2007 and AzTEC, Scott et al. 2008). GALEX has observed the central field deeply (Zamojski et al. 2007) and is currently monitoring this area. The Ultra-VISTA survey will undertake a deep $y J H K$ survey of the central $1.5 \mathrm{deg}^{2}$, half of which will be surveyed to the unprecedented limits of 26 AB mag (Arnaboldi et al. 2007).

By going for large area rather than extreme depth, most of the C-COSMOS sources are sufficiently bright to be detected in the rest of the pan-chromatic COSMOS data set, allowing rapid identifications (F. Civano et al. 2009, in preparation) and determination of their multiwavelength properties (e.g., M. Elvis et al. 2009, in preparation). On the other hand, C-COSMOS is sufficiently deep that significant numbers of normal and starburst galaxies with luminosity of $10^{42} \mathrm{erg} \mathrm{s}^{-1}$ can be detected up to $z \sim 0.9$, a redshift depth comparable with that of the galaxy redshift surveys in the COSMOS field (Taniguchi et al. 2007; Lilly et al. 2007). Adding the Chandra coverage to the COSMOS survey adds a valuable resource for the study of the co-evolution of black holes and their host galaxies, of the spectral energy distributions of faint quasars and active galactic nuclei, and the evolution of galaxies.

The summed image of the entire C-COSMOS field is shown in Figure 2, where colors have been mapped to X-ray bands.

This is the first of three papers presenting the basic results of the C-COSMOS survey over the whole field. Paper I (this paper) reports on the strategy, design, and execution of the C-COSMOS survey, and present the catalog of 1761 pointlike X-ray sources detected in C-COSMOS; Paper II (Puccetti et al. 2009) presents the details of the simulations carried out to optimize the source detection method; Paper III (F. Civano et al. 2009, in preparation) presents the identification of the X-ray sources with optical and infrared counterparts. We conclude by listing the primary science objectives foreseen for the C-COSMOS data. Papers on several of these topics are in preparation.

We assume a $\Lambda$ CDM cosmology with $\mathrm{H}_{0}=70 \mathrm{~km} \mathrm{~s}^{-1}$, $\Omega_{m}=0.27, \Omega_{\mathrm{vac}}=0.73$.

\section{THE CHANDRA COSMOS STRATEGY}

For C-COSMOS we have developed a strategy that uses $\sim 50 \%$ overlapping tiling of the $16.9 \times 16.9$ arcmin ACIS-I fields. This tiling produces a remarkably uniform sensitivity in the central part of the field, and a well-defined flux limit with a sharp cutoff (Figure 3; for details on the generation of sensitivity maps, see Section 7 in Paper II). This approach also ensures that the area with HPD $<2^{\prime \prime}$ is maximized, so that the unique Chandra high resolution imaging (van Speybroeck et al. 1997) can be exploited fully, albeit with $1 / 4$ of the exposure time. The good Chandra point-spread function (PSF) resolves sources $2^{\prime \prime}$ apart over $\sim 0.7 \mathrm{deg}^{2}$, corresponding to 8$16 \mathrm{kpc}$ separations for $z=0.3-0.9$, and locates point sources to $<4 \mathrm{kpc}$ at any redshift. Thus close mergers can be resolved, and nuclear sources distinguished from off-nuclear sources in galaxies (Ultra-luminous X-ray Sources, ULXs; Fabbiano 2006, Lehmer et al. 2006; Mainieri et al. 2009).

Point source detection sensitivities were estimated for three standard Chandra bands: soft (S, 0.5-2 keV), hard (H, 2$10 \mathrm{keV})$, and full (F, 0.5-10 keV). Due to the high background 
Table 1

C-COSMOS Flux Limits and Corresponding XMM-COSMOS Flux Limits

\begin{tabular}{lccc}
\hline \hline \multicolumn{1}{c}{ Band } & C-COSMOS $(\lim )^{\mathrm{a}}$ & C-COSMOS $(\log N-\log S)^{\mathrm{a}}$ & $X$ XMM-COSMOS \\
\hline Soft $(0.5-2 \mathrm{keV})$ & 1.9 & 2.5 & 5 \\
Hard $(2-10 \mathrm{keV})$ & 7.3 & 16 & 25 \\
Full $(0.5-10 \mathrm{keV})$ & 5.7 & $\ldots$ & $\ldots$ \\
\hline
\end{tabular}

Note.

${ }^{a}$ Flux limits are reported in units of $10^{-16} \mathrm{erg}^{-2} \mathrm{~s}^{-1}$, for bands up to $10 \mathrm{keV}$, but were measured only up to $7 \mathrm{keV}$. (See text for details.)

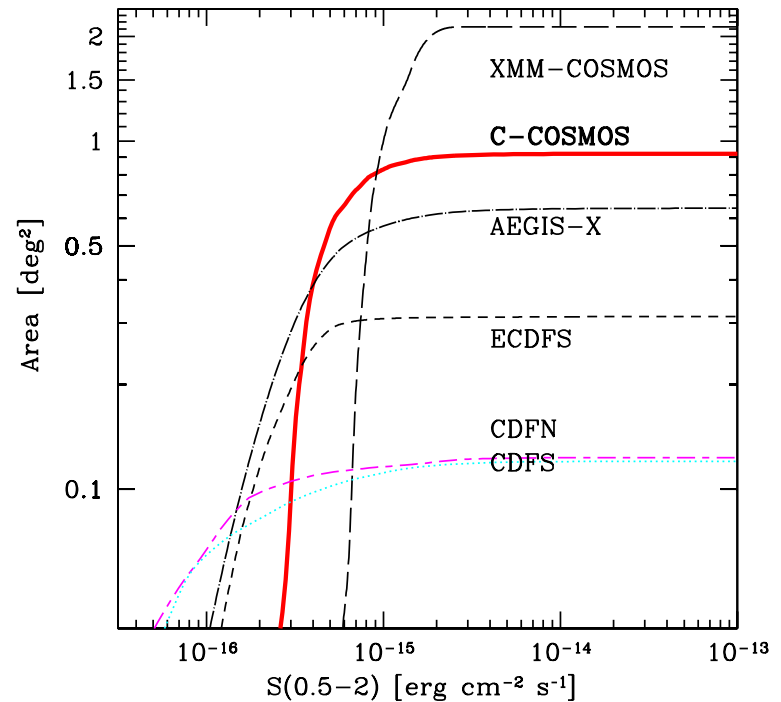

Figure 3. Area-flux curve for C-COSMOS (red solid line; $0.5-2 \mathrm{keV}$ ). The coverage of ECDFS (Lehmer et al. 2005; dashed line), AEGIS-X (Laird et al. 2008; dash-dotted line), CDFN (Alexander et al. 2003; magenta short-long dashed line), CDFS (Luo et al. 2008; cyan dotted line), and XMM-COSMOS (Cappelluti et al. 2009; black dashed line) are shown for comparison.

(A color version of this figure is available in the online journal.)

in the $7-10 \mathrm{keV}$ energy range, ${ }^{33}$ channels above $7 \mathrm{keV}$ were not used for source detection. (See Section 4.2.2 and Paper II for details). The C-COSMOS flux limits in three bands are reported in Table 1, together with the XMM-COSMOS limits for comparison: C-COSMOS sensitivity is three times below the corresponding flux limits for the XMM-COSMOS survey (dashed line; Cappelluti et al. 2009), making them complementary surveys.

The achieved sensitivity-area curve ${ }^{34}$ (Figure 3 ) has a sharp cutoff at low fluxes.

The C-COSMOS soft band flux limit corresponds to luminosities of $(0.8,4,11) \times 10^{41} \mathrm{erg} \mathrm{s}^{-1}$ at $z=(0.3,0.6,0.9)$ respectively, while the hard band flux limit corresponds to four times higher luminosities. Both luminous elliptical galaxies and starbursts often exceed these luminosities, and starburst galaxies are known to become common (Hornschemeier et al. 2003) at these X-ray fluxes.

The low ACIS background enables stacking analysis, in which counts at the positions of known classes of objects, e.g. subsets of the thousands of galaxies with redshifts, are co-added to increase the effective exposure time (Brusa et al. 2002; Hornschemeier et al. 2002; Brandt et al. 2001; Nandra et al. 2002; Fiore et al. 2008).

\footnotetext{
33 http://cxc.harvard.edu/contrib/maxim/bg/index.html\#spec

34 This curve is remarkably close to the predictions from the proposal, reflecting the high accuracy with which the requested tiling was executed.
}

Table 2

Coordinates of the C-COSMOS Field, Center, and Corners of the Outer and Inner Regions, Clockwise from the NE (Top Left)

\begin{tabular}{lc}
\hline \hline \multicolumn{1}{c}{ R.A. } & Decl. \\
\hline Center & \\
$10^{\mathrm{h}} 00^{\mathrm{m}} 24^{\mathrm{s}}$ & $+02^{\circ} 10^{\prime} 55^{\prime \prime}$ \\
\hline Outer region & \\
$10^{\mathrm{h}} 02^{\mathrm{m}} 45^{\mathrm{s}}$ & $+02^{\circ} 26^{\prime} 47^{\prime \prime}$ \\
$09^{\mathrm{h}} 59^{\mathrm{m}} 11^{\mathrm{s}}$ & $+02^{\circ} 46^{\prime} 45^{\prime \prime}$ \\
$09^{\mathrm{h}} 57^{\mathrm{m}} 54^{\mathrm{s}}$ & $+01^{\circ} 53^{\prime} 00^{\prime \prime}$ \\
$10^{\mathrm{h}} 01^{\mathrm{m}} 23^{\mathrm{s}}$ & $+01^{\circ} 33^{\prime} 59^{\prime \prime}$ \\
\hline Inner region & \\
$10^{\mathrm{h}} 02^{\mathrm{m}} 05^{\mathrm{s}}$ & $+02^{\circ} 21^{\prime} 13^{\prime \prime}$ \\
$09^{\mathrm{h}} 59^{\mathrm{m}} 30^{\mathrm{s}}$ & $+02^{\circ} 35^{\prime} 47^{\prime \prime}$ \\
$09^{\mathrm{h}} 58^{\mathrm{m}} 35^{\mathrm{s}}$ & $+01^{\circ} 59^{\prime} 19^{\prime \prime}$ \\
$10^{\mathrm{h}} 01^{\mathrm{m}} 11^{\mathrm{s}}$ & $+01^{\circ} 44^{\prime} 37^{\prime \prime}$ \\
\hline
\end{tabular}

\subsection{Design}

The C-COSMOS tiling scheme (Figure 4, left panel) covers the central area of the COSMOS field in the most efficient manner that we could devise. A $6 \times 6$ raster array of 36 ACIS-I pointings (one ACIS pointing field of view is outlined in black in Figure 4, left), each of $50 \mathrm{ks}$ nominal exposure, were chosen. The center of the array (Table 2) is slightly offset from the center of the COSMOS field to match the $z$-COSMOS deep field (Lilly et al. 2007).

The value of the 8.0 offset between pointing centers was chosen to be slightly less than the 8.3 size of an ACIS chip (Garmire et al., 2003; Chandra Proposers' Observatory Guide, aka POG, $2007^{35}$; Table 6.1), so that chip gaps are not co-added to create small scale dips in the effective exposure time.

The inner part of the field was covered by four exposures, to give a total nominal exposure of $200 \mathrm{ks}$ (effective exposure $\sim 160 \mathrm{ks})$ over a $42^{\prime} \times 42^{\prime}$ area $\left(0.5 \mathrm{deg}^{2}\right.$, green area in Figure 4$)$. The outer region has been covered by two observations (blue area) and the four corners covered by 1 observation (purple area). The corners of the outer and inner regions are reported in Table 2 clockwise from the top left.

Sources at a flux of $\sim 2.0 \times 10^{-16} \mathrm{erg} \mathrm{cm}^{-2} \mathrm{~s}^{-1}(0.5-2 \mathrm{keV})$ have a total of 5-10 summed counts in the four exposures, ensuring a good detection, given the low Chandra/ACIS background of $\sim 2$ counts/200 ks over a 2 arcsec radius circle (see Section 4.2.1).

The heavily overlapped tiling scheme produces a smooth exposure map that is flat to $12 \%$ in the central region (see Figure 4, right panel and Section 4.2.2).

\subsection{Comparison with Other Legacy Surveys}

Chandra observing time has been dedicated to several large legacy surveys: CDF-S (Giacconi et al. 2002; Luo et al. 2008),

\footnotetext{
35 Chandra X-ray Center publication TD 403.00.010.
} 

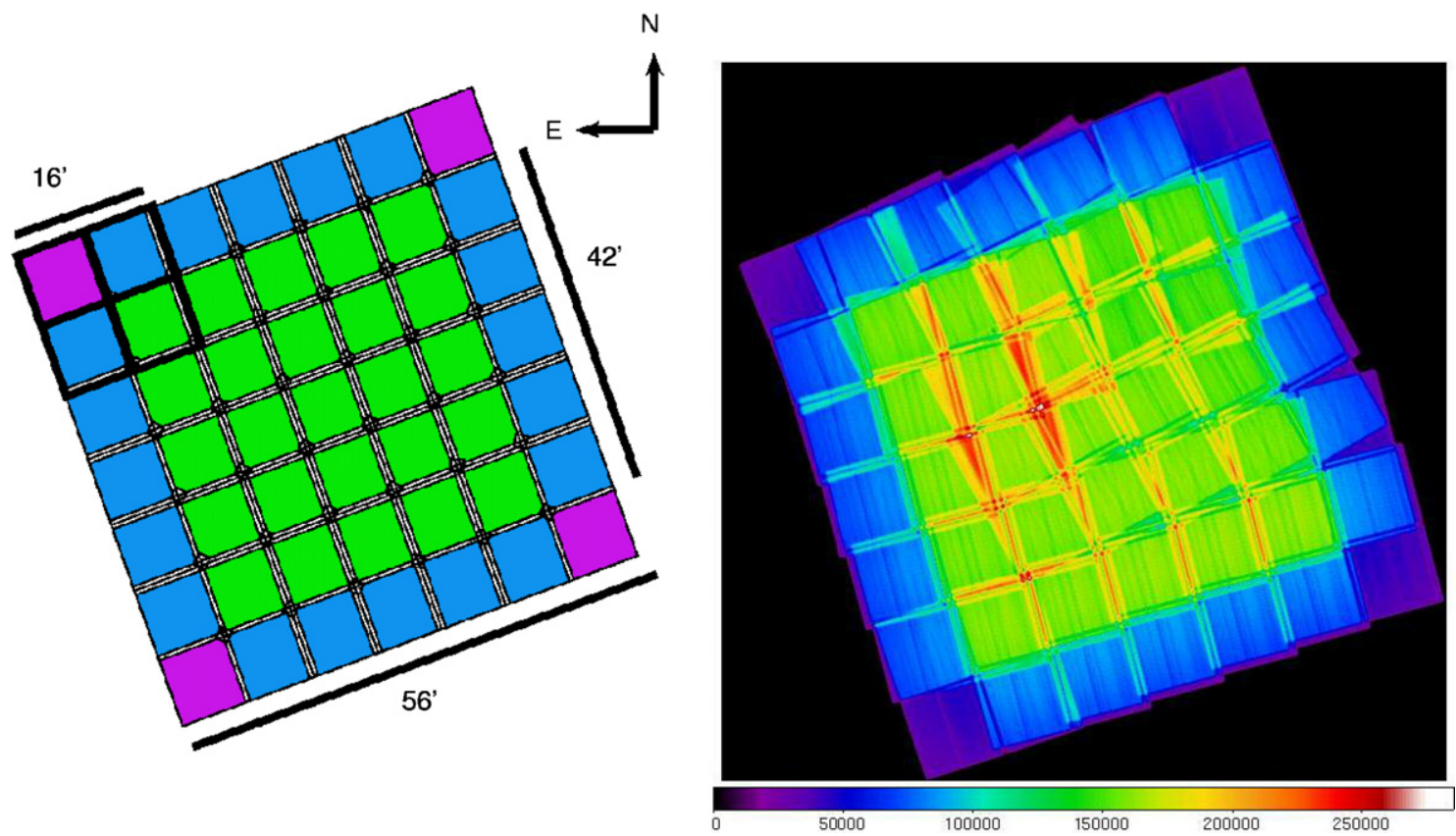

Figure 4. Left: the "as designed" C-COSMOS tiling for the 3650 ks pointings. The thick black box (top left) represents one ACIS-I pointing, the thin boxes all the pointings. Different colors show areas with different number of overlapping pointings: green -4 overlapping pointings; blue -2 overlapping pointings; purple -1 pointing. The black bars show roughly the relative dimensions of one pointing $\left(\sim 16^{\prime}\right)$, of the inner area with larger exposure $\left(\sim 42^{\prime}\right)$, and of the total field $\left(\sim 56^{\prime}\right)$. Raster point (see Table 3) 1-1 lies at the top right (NE) and 1-6 lies at the top left (NW). Right: the "as executed" exposure map for the C-COSMOS survey in the soft band. The color bar gives the achieved effective exposure in units of seconds.

CDF-N (Alexander et al. 2003), ECDF-S (Lehmer et al. 2005), AEGIS-X (Nandra et al. 2005; Laird et al. 2008), and XBootes (Murray et al. 2005). These surveys have different emphases in area and depth, so we summarize the special features of C-COSMOS here.

Like all contiguous area surveys, C-COSMOS has significant advantages over noncontiguous surveys (e.g., SEXSI, Harrison et al. 2003, Eckart et al. 2006; ChaMP, Kim et al. 2007), because of the difficulty of getting deep multiwavelength coverage of noncontiguous fields.

C-COSMOS is neither the deepest (CDFN and CDFS) nor the widest (XBootes) legacy Chandra survey. A comparable sensitivity has been reached in the somewhat smaller AEGIS field (the dot-dashed line in Figure 3; Laird et al. 2008). C-COSMOS differs from the other surveys by having the largest area at fluxes (3-10) $\times 10^{-16} \mathrm{erg} \mathrm{cm}^{-2} \mathrm{~s}^{-1}$ ( $S$ band), and a sharper low flux limit cutoff in the area surveyed than most other recent X-ray surveys. The single field CDF-S and CDF$\mathrm{N}$ have notably shallower roll-offs in their sensitivity curves (magenta and blue lines in Figure 3).

X-ray surveys are normally compared using an "area-depth" plot (e.g., Brandt \& Hasinger 2005). The differences in cutoff sharpness noted above require a slight revision of this plot to compare C-COSMOS with comparable contiguous Chandra and XMM-Newton surveys self-consistently. This is because the area normally quoted is the maximum area of the survey, while the normally quoted flux limit is that of the faintest source in the survey, which in most cases can be detected only in a much smaller area. If all surveys have similar cutoffs the difference is qualitatively unimportant, but in the case of C-COSMOS it makes a substantial difference.

We have used the area-flux curve of each survey to derive the flux at the point where each survey reaches $80 \%$ of the maximum survey area. We plot these values in Figure 5 (filled circles) for the Chandra contiguous area surveys (CDFN, Alexander et al. 2003; CDFS, Luo et al. 2008; ECDFS, Lehmer et al. 2005; AEGIS- X, Laird et al. 2008; XBootes, Murray et al. 2005; ELAIS-N, Manners et al. 2003), and for the $X M M$-Newton contiguous surveys that fill regions of the fluxarea plane (ELAIS-S1, Puccetti et al. 2006; XMM-COSMOS, Cappelluti et al. 2009; Lockman-Hole, Brunner et al. 2008). The C-COSMOS flux at $80 \%$ of the area covered $\left(0.72 \mathrm{deg}^{2}\right)$ in the soft band is $6 \times 10^{-16} \mathrm{erg} \mathrm{cm}^{-2} \mathrm{~s}^{-1}$.

Compared with other plots of this kind (e.g., Brandt \& Hasinger 2005) the points in Figure 5 tend to be moved diagonally toward smaller area and high flux limits. This shift can be quite large for surveys with shallow slopes at low fluxes in their area-flux limit curves (such as, for example, the deep fields).

Curves of constant numbers of sources (for the soft band) are shown in Figure 5 following the predictions of the Gilli et al. (2007) XRB model. ${ }^{36}$ The larger source numbers in XBootes and in the two COSMOS surveys are notable. Some soft band 1000 sources are predicted for C-COSMOS above the " $80 \%$ area" flux limit, while 1023 are actually detected.

The total number of sources in the CDF fields is, of course, significantly larger than the " $80 \%$ area" number The more sensitive, smaller area, parts of each survey add more sources than indicated by the dashed black lines, especially for the curves that have shallow cutoffs. To clarify this quite important point, Figure 5 also shows the area-flux curves for each survey down to their "20\% area" value. These curves show the differences between the surveys' flux limits. The differences between the two CDF deep fields are quite striking and are due to the slightly different observational strategies used for the two surveys (changing only the roll angle (CDF-N), or also moving the field center (CDF-S)). The figure caption gives both the $80 \%$ area

\footnotetext{
36 The curves have been computed using the tool "POrtable Multi Purpose Application for XRB and AGN counts" available at the Web site

http://www.bo.astro.it/ gilli/counts.html. We have checked that the numbers in each survey at the quoted $80 \%$ area flux levels agree with these curves.
} 


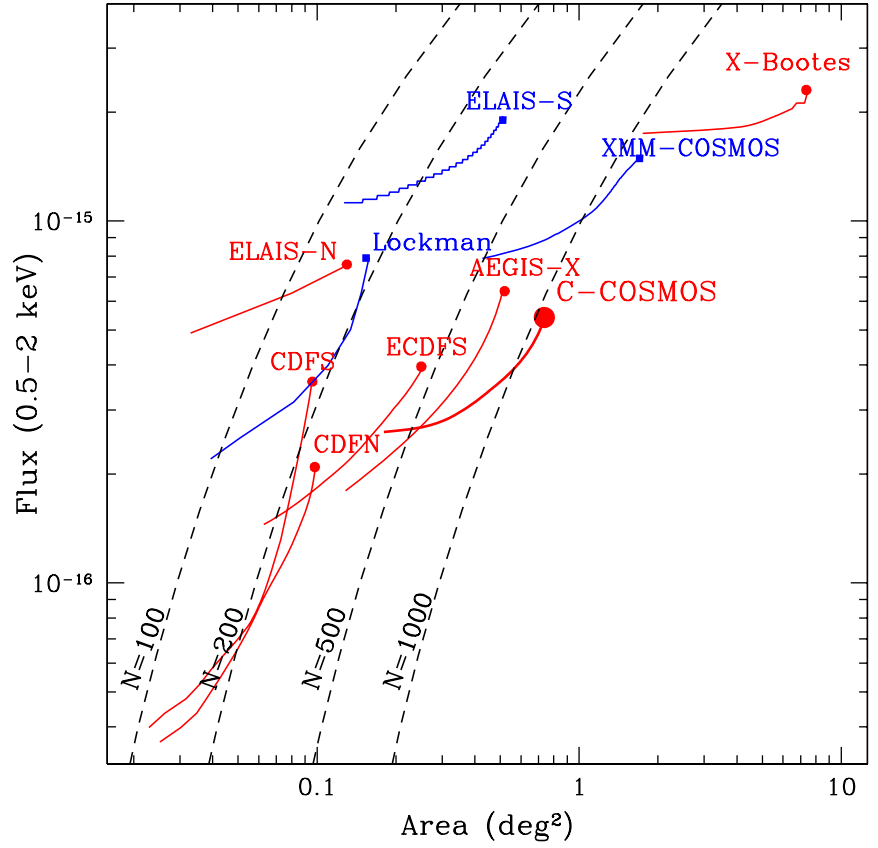

Figure 5. Area-flux curves for Chandra (red) and XMM-Newton (blue) contiguous X-ray surveys. Each survey has been plotted using each sensitivity curve starting from the flux corresponding to the area that is $80 \%$ of the maximum area for that survey (large points at the top of each curve), to the flux corresponding to the $20 \%$ of the total area (bottom of each curve). Data were taken from the following Chandra references: XBootes $(N(80)=$ $3963, N($ total $)=3180)-$ Murray et al. 2005; $\operatorname{CDFN}(N(80)=221, N($ total $)=$ $451)$-Alexander et al. 2003; CDFS $(N(80)=184, N($ total $)=392)$ - Luo et al. 2008; ECDFS $(N(80)=411, N($ total $)=598)$-Lehmer et al. 2005; AEGIS-X $(N(80)=689, N($ total $)=1032)$-Laird et al. 2008; ELAIS-N $(N(80)=156, N($ total $)=182)$-Manners et al. 2003; and XMM-Newton references: $X M M-\operatorname{COSMOS}(N(80)=1200, N($ total $)=1621)$-Cappelluti et al. 2009; Lockman $(N(80)=195, N($ total $)=340)$-Brunner et al. 2008; ELAIS-S $(N(80)=319, N($ total $)=395)$-Puccetti et al. 2006, C-COSMOS $(N(80)=$ $1070, N($ total $)=1340)$ - this paper, where " $N(80)$ " is the number of $S$ band sources at the $80 \%$ area fluxl, and " $N$ (total)" is the total number of $S$-band sources in the survey. The black dashed curves show the total number of 0.5 $2 \mathrm{keV}$ sources expected based on the $\log N-\log S$ relation predicted by Gilli et al. (2007) at the $80 \%$ area point. Each survey contains more sources at fainter fluxes and from smaller areas. The more vertical the sensitivity curve, the more of these fainter sources will be found.

(A color version of this figure is available in the online journal.)

and the total number of soft band sources for each survey and shows how the different area-flux curves affect the number of sources. The AEGIS-X survey (Laird et al. 2008), for example, has about double the number of $S$-band sources predicted at the $80 \%$ point, as do the two CDF fields. Instead, C-COSMOS, with a flatter flux-area curve, has $\sim 30 \%$ more $S$-band sources in total than the $80 \%$ area number.

Each of these surveys has extended multiwavelength coverage, but C-COSMOS is the only deep and wide X-ray survey field selected for both existing deep multiwavelength coverage, and for future legacy value, due to the equatorial location of the COSMOS field. The AEGIS field $\left(\delta=+52^{\circ}\right)$, the CDF-N field $\left(\delta=+62^{\circ}\right)$, and the XBootes field $\left(\delta=+35^{\circ}\right)$ are all too northerly to be accessible by ALMA or the VLT. The COSMOS field was also selected to have low IR cirrus emission, and a lack of bright stars, X-ray or radio sources in the field to maximize multiwavelength coverage.

C-COSMOS and XMM-COSMOS complement one another by providing large samples of sources over a wide flux range (Figure 8), while sharing the same extensive multiwavelength data set. $X M M$-COSMOS provides a larger sample of extended sources, while C-COSMOS provides a larger sample of starburst and normal galaxies.

\section{OBSERVATIONS}

A summary of the Chandra ACIS-I C-COSMOS observations as carried out is given in Table 3. Primarily because of thermal constraints on spacecraft components (POG; Section 3.3.3), many of the $36 \mathrm{C}$-COSMOS pointings were scheduled as two or more separate ObsIDs, giving 49 C-COSMOS observations in all. The indices $X-Y$ (1-1 through 6-6) describe the field numbers, where $X$ is an index in R.A. and $Y$ an index in decl., with $1-1$ being in the top right (NE) corner of Figure 4 (left panel), and 1-6 being in the top left (NW) corner.

The observations took place in two main blocks: 2006 December-2007 January and 2007 April-June (Table 3). The fields were observed at nominal roll angles of $250 / 70 \mathrm{deg}$, where the visibility of the COSMOS field is at maximum $(\sim 70 \%)$ and the pitch angle is such that the constraints are either unrestricted or restricted only to avoid overheating of the charged particle detector (EPHIN). As an equatorial field, the roll angle of the COSMOS field is quite stable (55.2-69.6, 248.4-256.2) for periods of $\sim 100$ days. As a result, the Chandra X-ray Center (CXC) Mission Planning team were able to maintain a tight roll angle range of $\pm 6^{\circ}$ around the nominal values (Table 3), leading to a highly uniform exposure of the whole field.

The mean effective exposure time per field (not per ObsID) is $46.3 \mathrm{ks}$, when only the good time intervals (GTIs), cleaned of the few high background times (Section 4.2.1), are used. ${ }^{37}$ The maximum exposure is $48.3 \mathrm{ks}$ and the minimum exposure (excluding a single $37.6 \mathrm{ks}$ exposure for field 2-5, Table 3 ) is $44.1 \mathrm{ks}$. So, with this exception, the range of exposures over the fully covered inner region varies by just $\pm 2.0 \mathrm{ks}(4 \%)$.

\section{DATA PROCESSING}

The data from the 49 obsids were uniformly processed in two phases using the CIAO 3.4 software tools $^{38}$ (Fruscione et al. 2006), the $\operatorname{yaxx}^{39}$ tool and custom versions of the XMM SAS detect tool EMLDETECT. ${ }^{40}$ Standard Level-1 and Level-2 processing pipeline ${ }^{41}$ (ASCDS version 7.6.9) from the $\mathrm{CXC}$ were used. In the first processing phase we determined astrometric corrections (see below) for each ObsID. These corrections were then applied in the second phase where we reprocessed all event data starting with Level-1 products.

Data processing involved the following series of steps, as summarized below:

1. Astrometric corrections $\left(<1^{\prime \prime} 1\right)$ to the standard COSMOS frame starting with the CXC supplied standard data products (Section 6);

2. Baseline data product creation by reprocessing all ObsIDs to a standard frame of reference using the new astrometry and standard CXC pipelines (Section 4.2);

3. Background reduction using high background time filtering (which affects only two ObsIDs) (Section 4.2.1);

\footnotetext{
37 This is $\sim 93 \%$ of the requested exposure, well within the $90 \%$ tolerance limit defined for Chandra scheduling.

$38 \mathrm{http}: / / \mathrm{cxc}$.harvard.edu/ciao/

39 http://cxc.harvard.edu/contrib/yaxx/

40 http://xmm.esac.esa.int/sas/8.0.0/EMLdetect

41 Pipeline processing levels are explained at

http://cxc.harvard.edu/ciao/data/sdp.html.
} 
Table 3

Chandra-COSMOS Observation Summary

\begin{tabular}{|c|c|c|c|c|c|c|}
\hline Obs. ID & Field & $\begin{array}{c}\text { Obs. Start } \\
\text { (UT) }\end{array}$ & $\begin{array}{c}\text { Exp. Time }{ }^{\mathrm{a}} \\
(\mathrm{ks})\end{array}$ & $\begin{array}{c}\text { R.A. } \\
(\mathrm{J} 2000.0)\end{array}$ & $\begin{array}{c}\text { Decl. } \\
(\mathrm{J} 2000.0)\end{array}$ & $\begin{array}{l}\text { Roll } \\
\text { (deg) }\end{array}$ \\
\hline 7995 & $1-1$ & 2007 Jun 01, 03:41 & 44.6 & 100202.05 & +022236.46 & 248.4 \\
\hline 7996 & $1-2$ & 2006 Dec $28,11: 28$ & 44.7 & 100131.99 & +022520.48 & 63.4 \\
\hline 7997 & $1-3$ & 2006 Dec 30, 21:10 & 44.5 & 100101.92 & +022804.50 & 62.8 \\
\hline 8494 & $1-4$ & 2006 Dec 16, 13:21 & 20.2 & 100031.85 & +023048.52 & 66.4 \\
\hline 8122 & $1-4$ & 2007 Jan 20, 10:15 & 28.0 & 100031.85 & +023048.52 & 55.2 \\
\hline 8493 & $1-5$ & 2006 Dec $12,18: 07$ & 19.3 & 100001.79 & +023332.55 & 66.4 \\
\hline 7998 & $1-5$ & 2007 Jan 10, 21:41 & 26.9 & 100001.79 & +023332.55 & 63.2 \\
\hline 8478 & $1-6$ & 2006 Nov $24,10: 17$ & 17.6 & 095931.72 & +023616.58 & 69.6 \\
\hline 7999 & $1-6$ & 2006 Nov $25,09: 24$ & 29.0 & 095931.72 & +023616.58 & 69.6 \\
\hline 8000 & $2-1$ & 2007 May 26, 20:23 & 45.2 & 100151.10 & +021505.52 & 253.2 \\
\hline 8001 & $2-2$ & 2007 Apr 02, 03:42 & 47.3 & 100121.03 & +021749.54 & 256.2 \\
\hline 8123 & $2-3$ & 2007 Apr 07, 13:40 & 48.3 & 100050.97 & +022033.55 & 255.2 \\
\hline 8002 & $2-4$ & 2006 Dec 19, 04:57 & 28.5 & 100020.90 & +022317.58 & 65.0 \\
\hline 8496 & $2-4$ & 2006 Dec $23,12: 05$ & 17.8 & 100020.90 & +022317.58 & 65.0 \\
\hline 8003 & $2-5$ & 2007 Apr 02, 17:53 & 37.6 & 095950.83 & +022601.61 & 255.2 \\
\hline 8004 & $2-6$ & 2006 Nov $27,02: 25$ & 15.3 & 095920.76 & +022845.64 & 68.6 \\
\hline 8482 & $2-6$ & 2006 Dec 02, 09:05 & 10.2 & 095920.76 & +022845.64 & 68.6 \\
\hline 8483 & $2-6$ & 2006 Dec 04, 03:02 & 21.3 & 095920.76 & +022845.64 & 68.6 \\
\hline 8005 & $3-1$ & 2007 Apr 25, 02:42 & 30.8 & 100140.15 & +020734.57 & 255.2 \\
\hline 8552 & $3-1$ & 2007 Apr 26, 09:33 & 14.4 & 100140.15 & +020734.57 & 255.2 \\
\hline 8124 & $3-2$ & 2007 Apr 08, 03:42 & 31.1 & 100110.08 & +021018.59 & 255.2 \\
\hline 8549 & $3-2$ & 2007 May 05, 17:17 & 17.2 & 100110.08 & +021018.59 & 255.2 \\
\hline 8503 & $3-3$ & 2006 Dec $31,10: 18$ & 20.0 & 100040.02 & +021302.61 & 62.2 \\
\hline 8006 & $3-3$ & $2007 \operatorname{Jan} 01,11: 48$ & 25.8 & 100040.02 & +021302.61 & 62.2 \\
\hline 8007 & $3-4$ & 2006 Dec 19, 22:18 & 21.1 & 100009.95 & +021546.64 & 64.2 \\
\hline 8497 & $3-4$ & 2006 Dec 25, 01:50 & 27.1 & 100009.95 & +021546.64 & 64.2 \\
\hline 8008 & $3-5$ & 2007 Jan 02, 04:39 & 45.0 & 095939.88 & +021830.67 & 61.9 \\
\hline 8009 & $3-6$ & 2007 Jan 02, 18:06 & 44.8 & 095909.81 & +022114.70 & 61.8 \\
\hline 8010 & $4-1$ & 2007 Apr 27, 18:45 & 32.9 & 100129.19 & +020003.29 & 255.2 \\
\hline 8553 & $4-1$ & 2007 Apr 29, 01:02 & 14.4 & 100129.19 & +020003.29 & 255.2 \\
\hline 8011 & $4-2$ & 2007 Apr 04, 04:08 & 45.8 & 100059.13 & +020247.30 & 255.2 \\
\hline 8012 & $4-3$ & 2007 Jan 04, 05:30 & 48.0 & 100029.06 & +020531.33 & 61.3 \\
\hline 8013 & $4-4$ & 2007 Jan 04, 19:44 & 46.9 & 095958.99 & +020815.36 & 61.1 \\
\hline 8014 & $4-5$ & 2007 Jan 05, 09:29 & 44.2 & 095928.92 & +021059.38 & 60.9 \\
\hline 8015 & $4-6$ & 2007 Jan 07, 09:53 & 44.1 & 095858.85 & +021343.42 & 60.2 \\
\hline 8550 & $5-1$ & 2007 Apr 18, 19:11 & 22.7 & 100118.25 & +015232.34 & 255.2 \\
\hline 8016 & $5-1$ & 2007 Apr 19, 20:24 & 23.3 & 100118.25 & +015232.34 & 255.2 \\
\hline 8017 & $5-2$ & 2007 Apr 04, 17:55 & 45.3 & 100048.18 & +015516.35 & 255.2 \\
\hline 8018 & $5-3$ & 2007 Apr 05, 07:17 & 45.8 & 100018.11 & +015800.38 & 255.2 \\
\hline 8019 & $5-4$ & 2007 Apr 06, 23:25 & 48.0 & 095948.04 & +020044.41 & 255.2 \\
\hline 8020 & $5-5$ & 2007 Apr 09, 06:12 & 47.8 & 095917.97 & +020328.44 & 255.2 \\
\hline 8021 & $5-6$ & 2007 Apr 09, 20:24 & 47.3 & 095847.90 & +020612.48 & 255.2 \\
\hline 8022 & $6-1$ & 2007 May 10, 23:28 & 30.9 & 100107.30 & +014501.39 & 251.4 \\
\hline 8555 & $6-1$ & 2007 May $12,16: 06$ & 16.2 & 100107.30 & +014501.39 & 251.4 \\
\hline 8023 & $6-2$ & 2007 Apr 10, 12:49 & 48.3 & 100037.24 & +014745.41 & 255.2 \\
\hline 8024 & $6-3$ & 2007 Apr 11, 21:40 & 47.9 & 100007.17 & +015029.44 & 255.2 \\
\hline 8025 & $6-4$ & 2007 Apr 12, 11:57 & 47.9 & 095937.10 & +015313.47 & 255.2 \\
\hline 8026 & $6-5$ & 2007 Apr 13, 07:31 & 45.8 & 095907.03 & +015557.49 & 255.2 \\
\hline 8027 & $6-6$ & 2007 Apr 14, 13:54 & 48.3 & 095836.96 & +015841.53 & 255.2 \\
\hline
\end{tabular}

Note.

${ }^{a}$ After GTI and high-background filtering for two affected obsids. Intervals of $8.50 \mathrm{ks}$ and $2.45 \mathrm{ks}$ (respectively) were eliminated from the two affected ObsIDs $(8003,8014)$.

4. Exposure map creation in the three energy bands F, S, and $\mathrm{H}$, using the standard CIAO tool sequence (Section 4.2.2);

5. Calculation of the sky coverage (i.e., the area covered to a given flux threshold) in the three energy bands, F, S, and H;

6. Candidate source detection using a wavelet technique (PWDeteCt; Damiani et al. 1997); ${ }^{42}$

\footnotetext{
42 We compared PWDETECT with the CIAO tool WAVDETECT used by most Chandra deep surveys on a subset of C-COSMOS fields, and found no substantive difference in the results; PWDETECT is a much faster algorithm, due to better memory buffering.
}

7. Selection of reliable sources, with a probability of being spurious $<2 \times 10^{-5}$ in at least one band, using maximum likelihood fitting (EMLDETECT) applied simultaneously to each $\mathrm{ObsID}$ at the positions of all candidate sources; Puccetti et al. (2009, Paper II) shows that EMLdETECT reconstructs the input count rate of simulations well, while both PWDetect and DETECTOR underestimate the input count rate by about $15 \%$;

8. Reliability checks for all sources using simulations, searches for outliers and visual checks (rejected 
candidate sources were all in the wings of bright source PSFs);

9. Aperture photometry of reliable sources. At high fluxes the systematic error in the PSF, which is intrinsic to the EMLDETECT method, becomes larger than the statistical error; this systematic error is not present for aperture photometry;

10. Derivation of reliability and completeness criteria for the source catalog, leading to a $\log N-\log S$ curve that provides an end-to-end check of the source extraction by comparing with other surveys in the same flux range (Section 9).

Steps 1-4 are discussed more fully in the following subsections. Complete details of the steps from 5 onwards, including details of the simulations and tests, are given in Paper II.

\subsection{Astrometry Corrections}

In the first phase we determined accurate astrometric offsets for each ObsID. The good absolute astrometry produced by Chandra (0'.6 at 90\% confidence, POG, Section 5) is still of the order of one ACIS pixel. To avoid a loss of sensitivity, correcting the astrometry to much less than one pixel error is needed before merging event files, or stacking.

To this end, we first produced a list of bright X-ray sources for each of the 49 ObsIDs, using the standard CIAO CELLDETECT tool. Starting with the standard ACIS Level-2 data products, we generated a broadband exposure map for each ACIS CCD using the CIAO ${ }^{43}$ tools ASPHIST, MKINSTMAP, and MKEXPMAP. These exposure maps and event files were then used as input to a Chandra-adapted version of the XMM-SAS tool EMLDETECT (see the next section), with an input source candidate catalog obtained by running the sliding cell detection tool EBOXDETECT with a high threshold. All sources detected with likelihood parameter $\mathcal{L}>10$ were compared with the CFHT MegaCam I-band catalog of the COSMOS field (Capak et al. 2007), selecting only the point-like sources with $I$ magnitudes in the range 18-23. Using this restricted magnitude range minimizes systematic effects introduced by bright stars (saturation) and faint background objects (misidentification), and is appropriate for sources in this flux range (Brandt \& Hasinger 2005). An optical-X-ray position correlation was computed using the likelihood algorithm included in the SAS task EPOSCORR (Cappelluti et al. 2007, 2009). This task uses all the possible counterparts of an X-ray source in the field to determine the most likely coordinate displacement. This method is independent of the actual spectroscopic identifications, but post facto all the identifications have proved to be correct (Paper III). No statistically significant offset in roll was required for any ObsID, so the change in roll was set to exactly zero. The systematic offsets between the X-ray and the optical positions were always smaller than 1.1 arcsec, with an average shift of $\Delta$ R.A. $=0.04$ and $\Delta$ decl. $=0.25$.

\subsection{Baseline Data Products}

The second phase of processing brought the 49 Chandra ObsIDs to a common reference frame using the offsets derived above, and generated the baseline data products that were then used as the starting point in all subsequent C-COSMOS analysis.

This processing was based on the CIAO thread for creating a new Level-2 event file from Level-1 products. ${ }^{44}$ First, a new

\footnotetext{
43 http://cxc.harvard.edu/ciao/

44 http://cxc.harvard.edu/ciao3.4/threads/createL2/
}

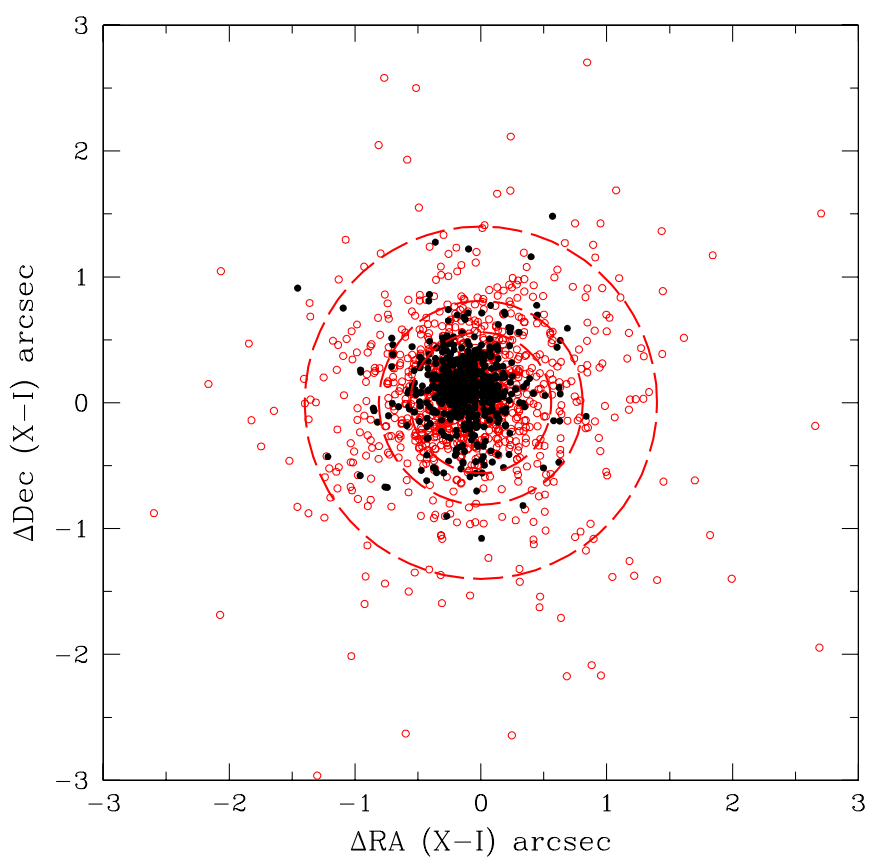

Figure 6. $X$-ray to $I$-band offsets ( $\Delta$ R.A., $\Delta$ decl.) in arcsec for $X$-ray sources with a secure identification (F. Civano et al. 2009, in preparation) after the aspect correction described in the text has been applied (Section 4.1). The circles encompass $68 \%\left(0^{\prime \prime} .56\right), 90 \%\left(0^{\prime \prime} .81\right)$, and $95 \%\left(1^{\prime \prime} .41\right)$ of the sources with optical counterparts and secure identification. Red dots mark sources with less than 50 counts in the full band.

(A color version of this figure is available in the online journal.)

aspect solution for each ObsID was generated to remove the astrometric offset for each ObsID derived in above section, using the REPROJECT_ASPECT tool. Then a new bad pixel file was created using ACIS_RUN_HOTPIX (see "background reduction" below). Finally, a new ACIS Level- 2 event file was then created for each ObsID using the ACIS_PROCESS_EVENTS tool, with (1) the standard ASCA grade set (grades [0, 2, 3, 4, and 6], POG Section 6.14), (2) pixel randomization turned off, (3) PHA randomization turned on, (4) very-faint mode processing enabled, and (5) the new aspect solution applied.

The astrometric corrections were checked using X-ray sources with point-like optical counterparts (F. Civano et al. 2009 , in preparation, Paper III) that were not used to derive the offsets for the individual ObsIDs. The residual systematic shift (X-ray - optical position) is on average $\Delta \alpha=-0^{\prime \prime} .1$ and $\Delta \mathrm{dec}=0^{\prime} .08$, and the $1 \sigma$ dispersion is $0^{\prime \prime} .56$ (i.e., the radius within which $68 \%$ of sources lie; Figure 6 ). We find that $90 \%$ of the X-ray positions agree with the identified optical/IR counterpart positions to within $1^{\prime \prime}$. 1 . The residual systematic shift is small enough that it will not affect the identification of any individual source and is smaller than the average $\mathrm{X}$-ray positional error, and therefore has not been used to correct the astrometry any further. The good quality of the data provides positions with subarcsecond accuracy at off-axis angle $<6^{\prime}$, in agreement with other Chandra surveys (0!23-1".90 in the CDFS, Luo et al. 2008; 0!3-1".67 in AEGIS, Laird et al. 2008).

\subsubsection{Background Reduction and Cosmic Ray Afterglow Detection}

Intervals of high background were determined by creating a background light curve for the ACIS-I CCD events with point sources found by WAVDETECT in the phase 1 processing removed. Only two obsids showed intervals with a significant 


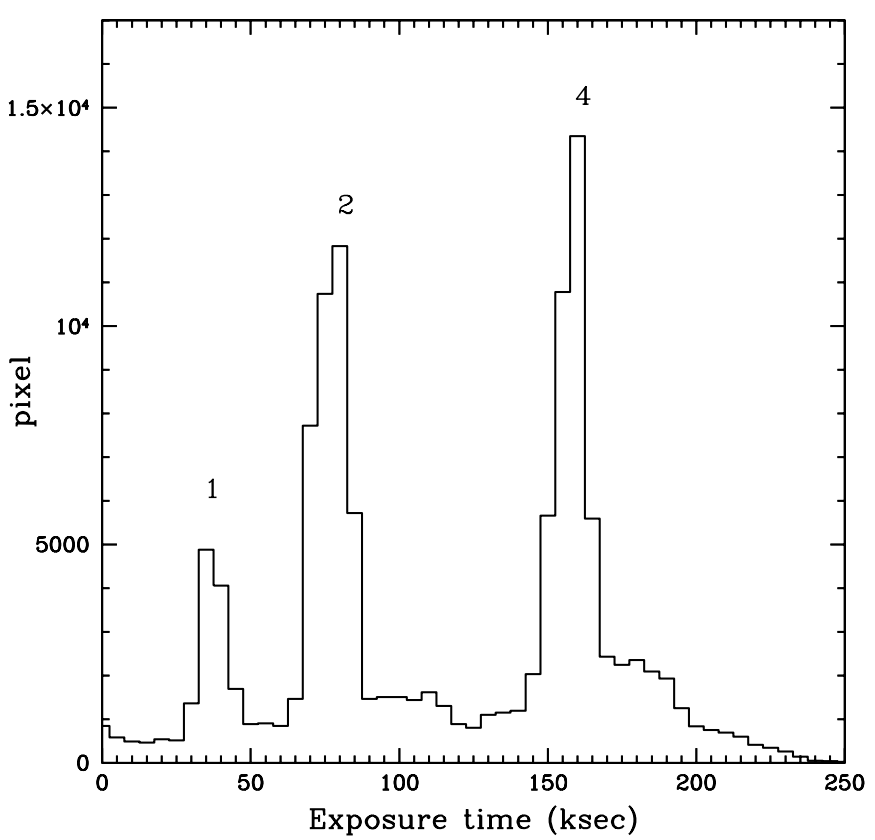

Figure 7. Histogram of the exposure times in the summed C-COSMOS field. The narrow peaks lie at the 1,2, and 4 exposure values. The broader bases correspond to overlaps caused by slight variations in the roll angles of the ObsIDs.

$(>5 \sigma)$ deviation from the quiescent background level (see Table 3).

Particular care was taken in the rejection of cosmic-ray afterglows. ${ }^{45}$ When a cosmic ray hits a CCD pixel a residual charge can remain localized for tens of seconds and produce "afterglow events," that appear to be X-ray events, at one location for several consecutive CCD frame readouts (POG Section 6.9). To reject cosmic ray afterglows we used the CIAO tool ACIS_RUN_HOTPIX ${ }^{46}$ and enabled VF mode background processing in ACIS_PROCESS_EVENTS. This process was successful as none of the C-COSMOS sources subsequently detected have the time localization characteristic of a spurious afterglow source. This procedure also gave a $25 \%-30 \%$ background reduction in the $0.5-7 \mathrm{keV}$ band.

The residual background is very stable over the full field of view at $\sim 1.8 \times 10^{-7}$ counts $\mathrm{s}^{-1}$ pixel $^{-1}$ or $\sim 2$ counts $/ 200 \mathrm{ks}$ over a 2 arcsec radius circle, which represents the typical size of our detection cell across the field. Following Alexander et al. (2003), in which the transition between a photon limited and a background limited regime is defined as $>3.3$ background counts per detection cell for $\mathrm{S} / \mathrm{N}=3$, we conclude that C-COSMOS is photon limited for point source detection.

\subsubsection{Exposure Maps and Sensitivity Curve}

We constructed exposure maps using the standard CIAO tool sequence of ASPHIST, MKINSTMAP, and MKEXPMAP, for each ObsID on a per-CCD basis, in each of three energy bands, S, H, F.

Figure 4 (right panel) shows a composite image of the effective exposure time (s) in the soft band. We clearly see the central region with four overlapping pointings, the side strips with two observations, and the corners covered by just one pointing. The uniformity of the exposure in the central
Table 4

Conversion Factors from Count Rates in the Soft, Full, and Hard Bands (0.5-2, $0.5-7,2-7 \mathrm{keV})$ to Fluxes in the Same Bands for Different Spectral Assumptions ${ }^{\mathrm{a}}$, Computed with the Chandra Cycle 8 Response Matrices

\begin{tabular}{lcc}
\hline \hline$\Gamma$ & $\begin{array}{c}\mathrm{N}_{H} \\
\left(\mathrm{~cm}^{-2}\right)\end{array}$ & Factor $^{\mathrm{b}}$ \\
\hline Soft Band & & \\
1.4 & Galactic & 1.87 \\
1.7 & Galactic & 1.81 \\
2.0 & Galactic & 1.75 \\
1.4 & $10^{22}$ & 2.12 \\
2.0 & $10^{22}$ & 2.15 \\
\hline Full Band & & \\
1.4 & Galactic & 0.75 \\
1.7 & Galactic & 0.89 \\
2.0 & Galactic & 1.04 \\
1.4 & $10^{22}$ & 0.51 \\
2.0 & $10^{22}$ & 0.71 \\
\hline Hard Band & & \\
1.4 & Galactic & 0.38 \\
1.7 & Galactic & 0.43 \\
2.0 & Galactic & 0.47 \\
1.4 & $10^{22}$ & 0.36 \\
2.0 & $10^{22}$ & 0.45 \\
\hline
\end{tabular}

Notes.

${ }^{\mathrm{a}} \Gamma=1.4, N_{H}=$ Galactic used for catalog fluxes.

${ }^{\mathrm{b}}$ conversion factor $\mathrm{CF}$ where Flux $=B_{\text {rate }} /\left(C F * 10^{11}\right)$, in units of counts $\mathrm{erg}^{-1} \mathrm{~cm}^{2}$.

region is shown by the histogram of the exposure times shown in Figure 7. This histogram shows narrow peaks at the 1,2, and 4 exposure values, which have Gaussian sigmas of 12.9, 13.6, and $19.3 \mathrm{ks}$, respectively, i.e., a $12 \%$ spread on the central region exposure. The total effective exposure in the inner, 4 exposure, region is $\sim 160 \mathrm{ks}$ at the peak, and $\sim 170 \mathrm{ks}$ at the mean, in the same region (see Figure 7).

The C-COSMOS sky coverage (i.e., the area covered as a function of limiting sensitivity) was computed in the three standard energy bands F, S, H using the exposure maps, the background maps and assuming a spectrum with $\Gamma=1.4$ and $\mathrm{N}_{H}=\mathrm{N}_{H}$ (Galactic). The sky coverage in the soft band is shown in Figure 3. More details on the full band and hard band are given in Paper II (Section 7).

The main uncertainty in the estimated sky coverage comes from the range of conversion factors from count rates to fluxes induced by the variety of intrinsic X-ray spectra in the X-ray population, in both power-law slope and intrinsic absorption, at a minimum. More complex spectra are surely present. An additional complication is that the average spectral properties are a function of the observed flux (Brandt \& Hasinger 2005). To estimate this uncertainty, we calculated the sky coverage for power-law spectra with $\Gamma=1.4$ and 2.0 with Galactic $\mathrm{N}_{H}$, and for absorbed power-law spectra with $\Gamma=1.4$ and 2.0 and $\mathrm{N}_{H}=10^{22} \mathrm{~cm}^{-2}$. The range of conversion factors, given by PIMMS, ${ }^{47}$ is a factor 2.0 in the $\mathrm{F}$ band, 1.3 in the $\mathrm{H}$ band and 1.2 in the $\mathrm{S}$ band (Table 4). As expected from the large width of the full band, the uncertainty for the full band is larger than for the soft and hard bands.

\footnotetext{
${ }_{47}$ http://cxc.harvard.edu/toolkit/pimms.jsp
}

$45 \mathrm{http}: / /$ cxc.harvard.edu/ciao/why/afterglows.html
$46 \mathrm{http} / /$ cxc.harvard.edu/ciao3.4/ahelp/acis_run_hotpix.html 
Table 5

Number of Sources Detected in Each Band at the Two Adopted Thresholds

\begin{tabular}{lcc}
\hline \hline Band & detml $\geqslant 10.8$ & $6<$ detml $<10.8$ \\
\hline Full $(\mathrm{F})$ & 1655 & 71 \\
Soft $(\mathrm{S})$ & 1340 & 88 \\
Hard $(\mathrm{H})$ & 1017 & 165 \\
\hline
\end{tabular}

Table 6

Number of Sources with detml $\geqslant 10.8$ in at Least One Band

\begin{tabular}{lc}
\hline \hline Bands & Number of Sources \\
\hline $\mathrm{F}+\mathrm{S}+\mathrm{H}$ & 922 \\
$\mathrm{~F}+\mathrm{S}$ & 474 \\
$\mathrm{~F}+\mathrm{H}$ & 257 \\
$\mathrm{~F}$ & 73 \\
$\mathrm{~S}$ & 32 \\
$\mathrm{H}$ & 3 \\
Total & 1761 \\
\hline
\end{tabular}

\section{POINT SOURCE CATALOG}

\subsection{Overview}

In this catalog we report the 1761 sources detected down to a defined threshold in at least one band. The threshold was chosen to balance completeness (the fraction of true sources detected) against reliability (the fraction of false sources detected). Paper II describes simulations that allowed us to choose a threshold which has a known completeness and reliability. We chose a probability threshold of $P=2 \times 10^{-5}$, giving $99.8 \%$ reliability for sources with more than 12 counts and $99.7 \%$ reliability for sources with 7 counts. This implies $\sim 3-5$ spurious F-band detections in the full field with $>12$ counts and 5 spurious detections with $>7$. At this threshold, the simulations then show that C-COSMOS is $87.5 \%$ complete for 12 count sources and $68 \%$ complete for 7 count sources. The C-COSMOS false source rates are consistent with those of other surveys (e.g., AEGIS-X, Laird et al. 2008) once the higher C-COSMOS threshold and larger average source extraction region are taken into account (see Paper II, Sections 6, 8, and 9).

The maximum likelihood statistic detml $=-\ln (P)=10.8$ for $P=2 \times 10^{-5}$, and this threshold detml was applied in EMLDETECT. The numbers of source detections at or above detml $=10.8$ are listed in the left column of Table 5. Crossmatching the sources with detml $>10.8$ in the three bands gives a total of 1761 sources. There are numerous sources with detml $>10.8$ in fewer than three bands. In these cases we can search for significant flux in the other bands to a 100 times higher $P$, as the area being searched is now 100 times smaller than the whole survey area (for a $5^{\prime \prime}$ cross-match radius). This corresponds to a threshold detml $=6$. In the right-hand column of Table 5 we give the numbers of sources detected in each band having $6<$ detml $<10.8$. Table 6 reports the numbers of catalog sources at or above detml $=10.8$ in three bands, two bands, or in only one band. (In this last case the sources must have detml $>10.8$ in order to have been selected at all.)

Almost a thousand (946) XMM-COSMOS sources have also been observed by Chandra with an exposure larger than 30 ks (Cappelluti et al. 2009), and 876 are present in the CCOSMOS catalog. Only 70 sources are not present in the Chandra catalog, while $24 X M M$-COSMOS sources have been resolved into two separate sources (M. Brusa et al. 2009, in preparation; Paper III) due to the better Chandra PSF. Of the 70 sources not recovered by Chandra, more

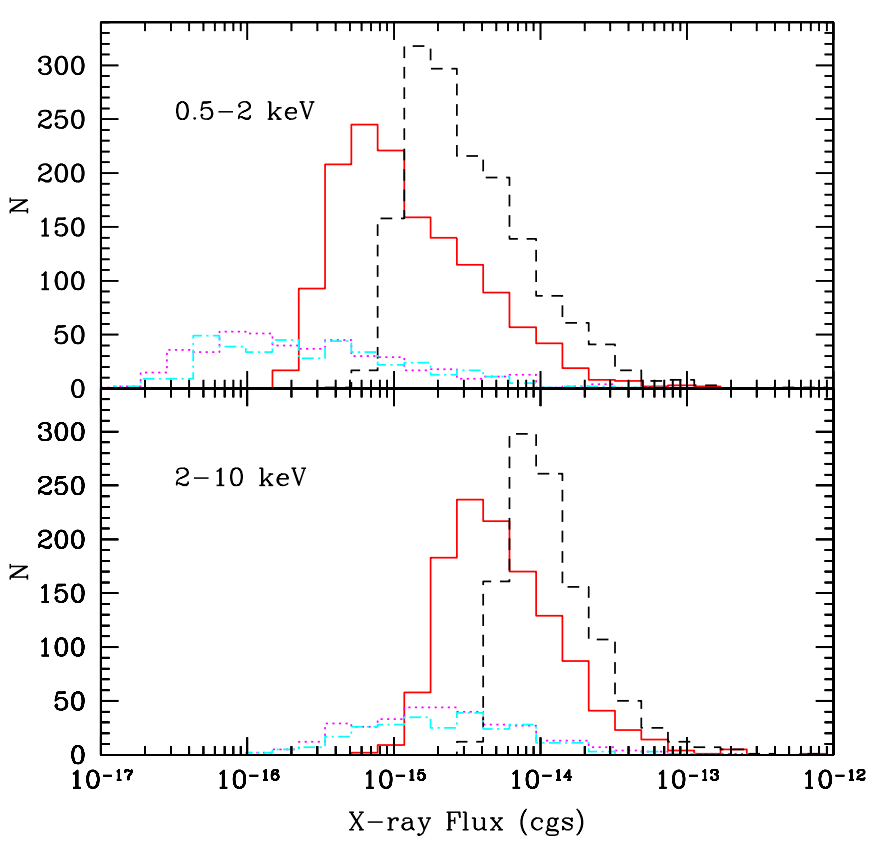

Figure 8. Distribution of fluxes for sources detected in the soft and hard band (continuous line) compared with the flux distribution of CDFN sources (dotted line), CDFS (dot-dashed line), and $X M M$-COSMOS sources (dashed line). Sources with upper limit have not been included in this figure.

(A color version of this figure is available in the online journal.)

than half are in regions with low exposure (between 30 and $50 \mathrm{ks}$ ) as, for example, in small gaps of low exposure (Figure 4). The remainder are either sources with only hard XMM detections or, after a visual inspection, they are found to be spurious $X M M$ sources, in agreement with the expected fraction of spurious sources. C-COSMOS and XMM-COSMOS combine to give a total of $\sim 2800$ unique COSMOS X-ray sources. The distribution of X-ray fluxes for the C-COSMOS sources in the soft and hard bands is shown in Figure 8. For comparison, we also show the flux distribution of CDFN (dotted line), CDFS (dotdashed line), and $X M M$-COSMOS detected sources (dashed line). The Chandra and XMM-Newton surveys are complementary in that, together, they span almost 3 orders of magnitude in $\mathrm{X}$-ray flux, and have over 100 soft band (and over 50 hard band) sources per 0.16 dex bin over about 1.5 orders of magnitude in flux. The well-defined cutoff in source numbers at faint fluxes, which reflects the tight exposure time distribution (Figure 7), is significantly different from the relatively flat distribution of CDFN (dotted line) and CDFS (dot-dashed line) source fluxes (Figure 8).

The complete catalog contains source positions and source count rates, exposure times, signal-to-noise ratio $(\mathrm{S} / \mathrm{N})$, and fluxes in the three bands and hardness ratios (see the next section). The catalog is ordered with the sources detected in the full band first, followed by those detected in the soft band only and by those detected in the hard band only.

The resulting catalog is available in the electronic version of the journal and on the "Chandra COSMOS Survey" Web site. ${ }^{48}$ Supporting data products (including images, event files and exposure maps) will be available at the "Chandra COSMOS Survey" Web site and at IRSA. ${ }^{49}$ At the Chandra COSMOS Survey it will also be possible to browse a database that includes

\footnotetext{
48 http://chandracosmos.cfa.harvard.edu/reports/analysis/20090310_TA_ source_catalog_2.1/

49 http://irsa.ipac.caltech.edu/data/COSMOS/
} 

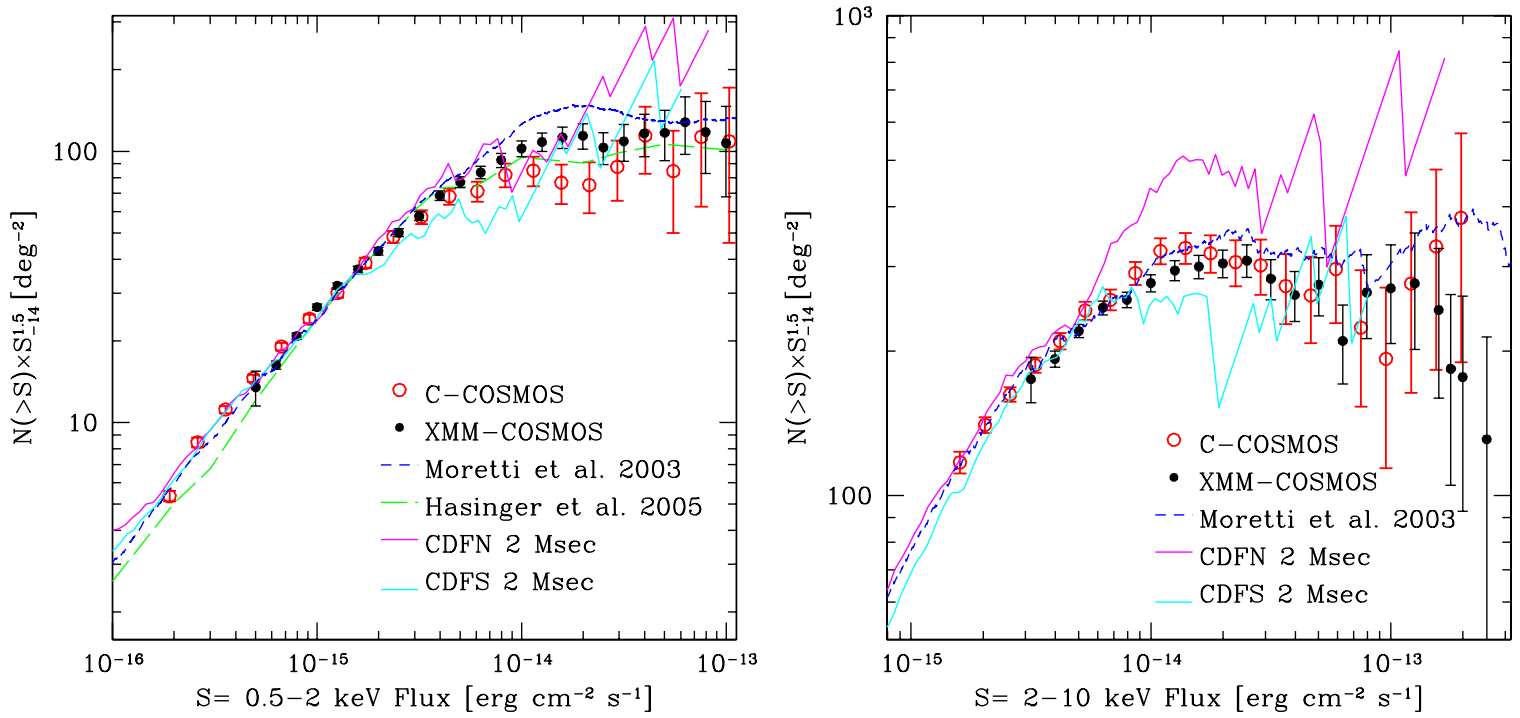

Figure 9. Euclidean-normalized, $\log N-\log S$ curves for C-COSMOS sources with detml $>10.8$ : left: soft band $(0.5-2 \mathrm{keV}$, red open circles), right: hard band (2-10 keV). The XMM-COSMOS curve (black filled circles, Cappelluti et al. 2009), the soft band curve of Hasinger et al. (2005; green line), the Moretti et al. (2003) compilation (blue dashed line), and the CDF-N (magenta solid line, Alexander et al. 2003) and CDF-S (cyan solid line, Luo et al. 2008) curves. The agreement is good over the flux interval where the various surveys have good statistics (see text).

"postage stamps" of the X-ray data for each source, along with the multiwavelength optical and infrared data, including the $I$ band, $K$-band, and Spitzer $3.6 \mu \mathrm{m}$ (Band 1) images used in Paper III to identify the sources.

\subsection{Catalog Description}

The EMLDETECT procedure was run on the three bands: soft, hard, and full. In order to be consistent with other results in literature, count rates estimated in the $2-7 \mathrm{keV}$ and $0.5-7 \mathrm{keV}$ energy bands were extrapolated into $2-10 \mathrm{keV}$ and $0.5-10 \mathrm{keV}$ fluxes, respectively, using a spectral slope, $\Gamma=1.4$. We also report the number counts obtained from aperture photometry (see Paper II).

Table 7 gives the columns of the catalog of the $1761 \mathrm{X}$-ray sources. A more detailed description of each column is reported below.

1. Column 1: Chandra source name, following the standard IAU convention with the prefix "CXOC" for "Chandra X-ray Observatory COSMOS" survey.

2. Column 2: source number. Sources are listed in order of detection: first those detected in the full band with detml $\geqslant 10.8$, followed by those detected in the soft band only and by those detected in the hard band only.

3. Columns 3-4: right ascension and declination in the $\mathbf{J} 2000$ coordinate system.

4. Column 5: positional error $\left(\sqrt{\sigma_{\text {R.A. }}^{2}+\sigma_{\text {decl. }}^{2}}\right)$ computed using the following equation $\operatorname{Pos}_{\text {error }}=\mathrm{PSF}_{\text {radius }} / \sqrt{S}$, where $S$ is the number of net source counts, after the subtraction of the background, in a circular region of radius corresponding to the $50 \%$ encircled energy in the field where the source is at the lowest off-axis angle (Paper II).

5. Column 6-7: count rate and count rate error in the full band (0.5-7 keV). These are effective count rates that would apply if the source had been observed at the aim point in every pointing, i.e., computed by dividing the best fit counts for each source by the effective exposure time at the position of each source (the effective exposure time includes
Table 7

Data Fields in the Catalog

\begin{tabular}{|c|c|c|}
\hline No. & Field & Note \\
\hline 1 & NAME & Chandra source name \\
\hline 2 & Source \# & source number. \\
\hline 3 & R.A. & Chandra Right Ascension (J2000, hms) \\
\hline 4 & DEC & Chandra Declination (J2000, dms) \\
\hline 5 & pos_err & Positional error [arcsec] \\
\hline 6 & f_rate & $0.5-7 \mathrm{keV}$ count rate (counts s$^{-1}$ ) \\
\hline 7 & f_rate_err & $0.5-7 \mathrm{keV}$ count rate error (counts s${ }^{-1}$ ) \\
\hline 8 & f_flux & $0.5-10 \mathrm{keV}$ Flux $\left[\mathrm{erg} \mathrm{cm}^{-2} \mathrm{~s}^{-1}\right.$ ] \\
\hline 9 & f_flux_err & $0.5-10 \mathrm{keV}$ Flux error [erg $\mathrm{cm}^{-2} \mathrm{~s}^{-1}$ ] \\
\hline 10 & f_snr & $0.5-7 \mathrm{keV} \mathrm{S} / \mathrm{N}$ Ratio \\
\hline 11 & f_exptime & $0.5-7 \mathrm{keV}$ exposure time $[\mathrm{ks}]$ \\
\hline 12 & f_cts_ap & $0.5-7 \mathrm{keV}$ aperture photometry net counts [counts] \\
\hline 13 & f cts ap err & $0.5-7 \mathrm{keV}$ aperture photometry net counts error [counts] \\
\hline 14 & f_exptime_ap & $0.5-7 \mathrm{keV}$ exposure time from aperture photometry [ks] \\
\hline 15 & S_rate & $0.5-2 \mathrm{keV}$ count rate (counts s $^{-1}$ ) \\
\hline 16 & s_rate_err & $0.5-2 \mathrm{keV}$ count rate error (counts s${ }^{-1}$ ) \\
\hline 17 & s flux & 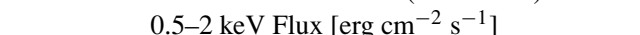 \\
\hline 18 & S_flux_err & $0.5-2 \mathrm{keV}$ Flux error [ $\mathrm{erg} \mathrm{cm}^{-2} \mathrm{~s}^{-1}$ ] \\
\hline 19 & s_snr & $0.5-2 \mathrm{keV}$ S/N Ratio \\
\hline 20 & s_exptime & $0.5-2 \mathrm{keV}$ exposure time $[\mathrm{ks}]$ \\
\hline 21 & s_cts_ap & $0.5-2 \mathrm{keV}$ aperture photometry net counts [counts] \\
\hline 22 & s_cts_ap_err & $0.5-2 \mathrm{keV}$ aperture photometry net counts error [counts] \\
\hline 23 & s_exptime_ap & $0.5-2 \mathrm{keV}$ exposure time from aperture photometry [ks] \\
\hline 24 & h_rate & $2-7 \mathrm{keV}$ count rate (counts $\mathrm{s}^{-1}$ ) \\
\hline 25 & h_rate_err & $2-7 \mathrm{keV}$ count rate error (counts $\mathrm{s}^{-1}$ ) \\
\hline 26 & h_flux & $2-10 \mathrm{keV}$ Flux $\left[\mathrm{erg} \mathrm{cm}^{-2} \mathrm{~s}^{-1}\right]$ \\
\hline 27 & h_flux_err & 2-10 keV Flux error [ $\mathrm{erg} \mathrm{cm}^{-2} \mathrm{~s}^{-1}$ ] \\
\hline 28 & h_snr & 2-7 keV S/N Ratio \\
\hline 29 & h_exptime & $2-7 \mathrm{keV}$ exposure time $[\mathrm{ks}]$ \\
\hline 30 & h cts ap & $2-7 \mathrm{keV}$ aperture photometry net counts [counts] \\
\hline 31 & h_cts_ap_err & $2-7 \mathrm{keV}$ aperture photometry net counts error [counts] \\
\hline 32 & h_exptime_ap & $2-7 \mathrm{keV}$ exposure time from aperture photometry [ks] \\
\hline 33 & $\mathrm{hr}$ & hardness ratio \\
\hline 34 & hr_lim_lo & hardness ratio $90 \%$ lower limit \\
\hline 35 & hr_lim_hi & hardness ratio $90 \%$ upper limit \\
\hline
\end{tabular}

Note. The C-COSMOS Bright Source Catalog is available in the electronic version of the journal. 
corrections for vignetting, dither, bad pixels and spatially dependent quantum efficiency). The count rate error at $68 \%$ confidence level was computed using the equation error $==\frac{\sqrt{C_{s, 90 \%}+(1+a) B_{90 \%}}}{0.9 \cdot T}$, where $C_{s}$ are the source counts estimated by EMLdetect, corrected to an area including $90 \%$ of the PSF, ${ }^{50} \mathrm{~B}$ are the background counts evaluated from the background rate (counts pixel $^{-1}$ ) estimated by EMLdetect multiplied for an area of radius $R_{w}$, which is the mean of the radii, corresponding to $90 \%$ enclosed counts fraction (ECF) of each observation, weighted by the observation exposure relative to the total exposure, and $T$ is the vignetting corrected exposure time at the position of the source from the exposure maps. We use $a=0.5$, to allow for uncertainties in the background, which is computed through the EMLdETECT procedure (see Paper II for more details).

6. Column 8-9: full band $0.5-10 \mathrm{keV}$ fluxes and errors were computed converting count rates to fluxes using the following formula: Flux $=B_{\text {rate }} /\left(C F * 10^{11}\right)$, where $B_{\text {rate }}$ is the count rate in each band as described in Column $6, \mathrm{CF}$ is the energy conversion factor 0.742 counts $\mathrm{erg}^{-1} \mathrm{~cm}^{2}$ (and 1.837 and 0.381 for the soft and hard, 2-10 keV band respectively) appropriate for a power-law spectrum with spectral index $\Gamma=1.4$ and Galactic column density $N_{H}=2.7 \times 10^{20} \mathrm{~cm}^{-2}$. For sources not detected in this band, a $90 \%$ upper limit is reported (see Paper II for details).

7. Column 10: full band signal-to-noise ratio.

8. Column 11: full band exposure time derived from the exposure map.

9. Column 12-13: the aperture photometry counts and error in the full band $(0.5-7 \mathrm{keV})$ are derived from event data for each individual Obsid and CCD where a source lands. Note that $\left(F \_\right.$rate $\left.\times f_{\text {_exptime }}\right) \neq f_{\_}$cts_ap. Circular extraction regions corresponding to the $90 \% \mathrm{ECF}$ for that observation are centered on the source R.A., decl. The individual photometry values are then merged to produce a single set of values accounting for the ECF for each ObsID, given the different extraction regions needed.

10. Column 14: exposure time (ks) from the same region used to generate the aperture photometry.

11. Column 15-23: same as Columns 6-14 for the soft band (0.5-2 keV).

12. Column 24-32: same as Columns 6-14 for the hard band (2-7 keV). Fluxes and errors are computed for the 2 $10 \mathrm{keV}$ band with the conversion factor quoted above.

13. Column 33-35: hardness ratio and 90\% upper and lower errors computed as follows: $\mathrm{H}-\mathrm{S} / \mathrm{H}+\mathrm{S}$, where $H$ are the counts in the hard band and $S$ the counts in the soft band. The hardness ratio was calculated starting with the EMLDETECT rate values. Upper and lower limits were calculated using the Bayesian estimation of hardness ratio code (BEHR; Park et al. 2006). Pseudo-source and background count values were generated using the net count rate, background rate (per pixel), and a 3 arcsec source aperture and 520 arcsec aperture for background areas. The aperture photometry was unsuitable for this purpose because the individual extraction apertures do not have the constant background/source area ratios required by the assumptions used in BEHR.

\footnotetext{
50 http://cxc.harvard.edu/caldb/
}

\subsection{Catalog Completeness and Number Counts}

In order to provide an end-to-end check that the many calibration steps taken in deriving the Chandra COSMOS point source catalog have been performed correctly, we constructed the observed $\log N-\log S$ curve, i.e. the number of sources, $N(>S)$, detected per square degree brighter than a given flux,

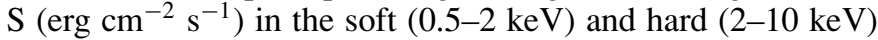
bands. Because at the limiting fluxes the sky coverage is small (Figure 3), and so has a large fractional error, we used the flux limits given in Table 1, Column 3, thus omitting the faintest $\sim 10$ sources. X-ray source counts in this flux and energy range have been well studied, giving us a good baseline against which to compare C-COSMOS (Cappelluti et al. 2009).

The results for sources detected at detml $>10.8$ (Table 5, left column) are shown in Figure 9, normalized by a Euclidean 1.5 slope to enable differences between various X-ray $\log N-$ $\log S$ curves to be seen easily. Figure 9 also shows comparisons with several other $\log N-\log S$ curves: from Moretti et al (2003, blue dashed line), which combines data from ROSAT (for bright sources), XMM-Newton (for intermediate flux sources), and Chandra for faint sources; from Hasinger et al. (2005) $\log N-$ $\log S$ (green dashed line); and from the CDF-N (magenta solid line; Alexander et al. 2003) and CDF-S (cyan solid line; Luo et al. 2008) curves. In the range where these curves overlap and $\mathrm{C}$-COSMOS has good statistics the agreement is excellent, and C-COSMOS extends a factor $\sim 4$ below the XMM-COSMOS limit, as expected.

In the soft band, around $\sim 2 \times 10^{-14} \mathrm{erg} \mathrm{cm}^{-2} \mathrm{~s}^{-1}$, the C-COSMOS $\log N-\log S$ shows a $\sim 20 \%-30 \%$ underdensity at a $2 \sigma$ level with respect to the XMM-COSMOS source counts. In order to evaluate this deviation, we estimated the amplitude of the fluctuations expected due to sample and cosmic variance. According to Yang et al. $(2004,2006)$ and Cappelluti et al. (2009), the fluctuations of the counts in a box of area $\Omega \mathrm{deg}^{2}$ of a population of $\mathcal{N} \mathrm{deg}^{-2}$ sources at a given flux limit is given by a linear combination of a Poisson fluctuations and a cosmic variance component introduced by source clustering:

$$
\sigma_{c v}^{2}=\mathcal{N}+\frac{\mathcal{N}}{\Omega^{2}} \int w(\theta) d \theta_{1} d \theta_{2}
$$

In Equation (1) $w(\theta)$ is the angular autocorrelation function expressed as a $w(\theta)={\frac{\theta}{\theta_{0}}}^{-\gamma}$. According to Cappelluti et al. (2007), Equation (1) can be solved analytically by knowing the slope and the amplitude of $w(\theta)$. By using the source surface density of soft X-ray sources at $2 \times 10^{-14} \mathrm{erg} \mathrm{cm}^{-2} \mathrm{~s}^{-1}$ (i.e., $\sim 30$ source $\mathrm{deg}^{-2}$ ) on a box of $0.9 \mathrm{deg}^{2}$, and assuming the angular autocorrelation function of Miyaji et al. (2007) for $X M M$-COSMOS (i.e., $\theta_{0}=2^{\prime \prime}, \gamma=1.8$ ), we determined $\sigma^{2} \sim 36$ which corresponds to a fraction variance of $20 \%$ of the source counts. We can therefore conclude that a deviation of the size observed can be introduced by a single structure, in an area of XMM-COSMOS not covered by Chandra, that generates a fluctuation in the bright source counts at $1.5 \sigma$ level.

Another check of the source detection efficiency at the brighter C-COSMOS flux levels is a comparison with the $X M M$ COSMOS survey (Hasinger et al. 2007). As shown by Cappelluti et al. (2009) and M. Brusa et al. (2009, in preparation), C-COSMOS recovers $\sim 93 \%$ of the XMM sources in the C-COSMOS field, resolving $\sim 3 \%$ into close pairs. 


\section{CONCLUSIONS AND FUTURE WORK}

We have presented the $\sim 0.9 \mathrm{deg}^{2}$ Chandra COSMOS survey (C-COSMOS) and a catalog of point sources from that survey. Employing a heavily overlapping tiling of ACIS-I observations has proven an effective method of covering a large area to a well-defined exposure $( \pm 12 \%)$ and uniform flux limit. The central $\sim 0.5 \mathrm{deg}^{2}$ achieved an exposure of $160 \mathrm{ks}$, and the outer $\sim 0.4 \mathrm{deg}^{2}$ achieved an exposure of $\sim 80 \mathrm{ks}$. The equatorial location of COSMOS helped to produce a uniform tiling pattern by allowing an almost constant roll angle for Chandra observations over most of the target visibility window. The point source catalog from the C-COSMOS survey has a flux limit of $2 \times 10^{-16} \mathrm{erg} \mathrm{cm}^{-2} \mathrm{~s}^{-1}(0.5-2 \mathrm{keV})$ and contains 1761 sources detected in at least one band with a probability of being spurious of $<2 \times 10^{-5}($ detml $\geqslant 10.8)$.

The novel three-stage source detection method employed (Paper II) coped well with the peculiarities of the C-COSMOS tiling scheme and, more generally, is good at separating close pairs of sources, while retaining photometric accuracy. The C-COSMOS sky coverage has a sharp cutoff which produces a homogeneous flux threshold over the whole area and the soft band $\log N-\log S$ curve for C-COSMOS matches well the Hasinger et al. (2005) determination over a broad flux range, giving us high confidence in the completeness of the catalog down to the limiting flux.

The catalog is available in the electronic version of the journal and on the "Chandra COSMOS Survey" Web site ${ }^{48}$ Supporting data products (including images, event files and exposure maps) are available at the "Chandra COSMOS Survey" Web site and at IRSA. ${ }^{49}$

The subarcsecond accuracy of the Chandra positions, together with the rich pre-existing deep multiwavelength coverage of the COSMOS field, allows us to reach a $96 \%$ identification rate for the C-COSMOS sources with counterparts in both optical and infrared, and $99.7 \%$ in at least one band (Paper III).

A parallel effort on the detection of extended sources in the C-COSMOS field finds $\sim 50$ groups and clusters (A. Finoguenov et al. 2009, in preparation).

We anticipate a rich haul of science results from C-COSMOS. The Chandra sources have already resolved ambiguous source identifications from the XMM-COSMOS survey (Hasinger et al. 2007, Brusa et al. 2007, 2008, Cappelluti et al. 2007, 2009). The paper by Fiore et al. (2008) on the stacking analysis of sources with extreme mid-infrared-to-optical ratio, presumably Compton-thick AGNs, has been recently accepted. Several further papers are in preparation or submitted on off-nuclear sources in galaxies (Mainieri et al. 2009), X-ray source correlation functions (Miyaji et al. 2007), the three-dimensional cluster/AGN cross-correlation function (Cappelluti et al. 2009), high X-ray/optical flux ratio objects (F. Civano et al. 2009, in preparation), high-redshift QSO (F. Civano et al. 2009, in preparation), and other topics.

A basic X-ray spectral analysis of the nearly 500 sources with more than 80 counts ( $\sim 23 \%$ of the total sample) becomes possible. The resulting spectral slopes and absorbing column densities will allow the statistical properties of a large sample at substantial redshift and over a uniform and contiguous field to be studied effectively (G. Lanzuizi et al. 2009, in preparation).

There is information in C-COSMOS below the current catalog flux limit, thanks to the low background of Chandra ACIS. A "stacking" analysis (Brusa et al. 2002; Hornschemeier et al. 2002,2003 ) allows the mean X-ray properties of groups of objects to be determined. Miyaji et al. (2007) have solved the issues created by the C-COSMOS tiling scheme for stacking and papers using this tool are in preparation on $z \sim 1$ elliptical galaxies (Kim et al. 2007). The potential uses of stacking in the C-COSMOS field are extensive, thanks to the multiple data sets available from which to choose samples for stacking. For example, there will be $\sim 2 \times 10^{4}$ galaxies with good optical spectra from $z$-COSMOS (Lilly et al. 2007) in the C-COSMOS field. This entire sample is well characterized both morphologically via $H S T$ imaging, and in terms of stellar population, from the UV to far-IR coverage of the other telescopes that have observed COSMOS (Scoville et al. 2007b). This rich data set will enable galaxy X-ray evolution studies by environment, morphology, and luminosity using fine-grained stacks of C-COSMOS data with $\sim 100$ galaxies per bin, for an effective exposure time of $\sim 20 \mathrm{Ms}$ per bin.

Clearly the C-COSMOS survey will be of value for some time.

We thank the Chandra mission planning team, especially Pat Slane and Jan Vrtilek, and the Chandra pipeline data processing team for the extraordinary efforts they put into the successful scheduling and execution of C-COSMOS. We also thank the rest of COSMOS team whose support has been invaluable in reaching this stage. We thank Bin Luo for sensitivity curves of Chandra Deep Fields. We gratefully thank the Chandra EPO team, in particular Eli Bressert, for creating the true color X-ray image. This research has made use of data obtained from the Chandra Data Archive and software provided by the Chandra $X$-ray Center (CXC) in the application packages CIAO and Sherpa.

This work was supported in part by NASA Chandra grant number GO7-8136A (M.E., C.V., M.B., A.F.), NASA contract NAS8-39073 (Chandra X-ray Center), and by NASA/ADP grant NNX07AT02G (TM at UCSD). In Italy this work is supported by ASI/INAF contracts I/023/05/0, I/024/05/0 and I/088/06, by PRIN/MUR grant 2006-02-5203. In Germany this project is supported by the Bundesministerium für Bildung und Forschung/Deutsches Zentrum für Luft und Raumfahrt and the Max Planck Society.

\section{REFERENCES}

Alexander, D. M., et al. 2003, AJ, 126, 539

Arnaboldi, M., Neeser, M. J., Parker, L. C., Rosati, P., Lombardi, M., Dietrich, J. P., \& Hummel, W. 2007, Messenger, 127, 28

Bertoldi, F., et al. 2007, ApJS, 172, 132

Brandt, W. N., \& Hasinger, G. 2005, ARA\&A, 43, 827

Brandt, W. N., Hornschemeier, A. E., Schneider, D. P., Alexander, D. M., Bauer, F. E., Garmire, G. P., \& Vignali, C. 2001, ApJ, 558, L5

Brunner, H., Cappelluti, N., Hasinger, G., Barcons, X., Fabian, A. C., Mainieri, V., \& Szokoly, G. 2008, A\&A, 479, 283

Brusa, M., et al. 2002, ApJ, 581, 89

Brusa, M., et al. 2007, ApJS, 172, 353

Capak, P., et al. 2007, ApJS, 172, 99

Cappelluti, N., et al. 2007, ApJS, 172, 341

Cappelluti, N., et al. 2009, A\&A, 497, 635

Damiani, F., Maggio, A., Micela, G., \& Sciortino, S. 1997, ApJ, 483, 350

Dickey, J. M., \& Lockman, F. J. 1990, ARA\&A, 28, 215

Eckart, M. E., Stern, D., Helfand, D. J., Harrison, F. A., Mao, P. H., \& Yost, S. A. 2006, ApJS, 165, 19

Fabbiano, G. 2006, ARA\&A, 44, 323

Ferrarese, L., \& Merritt, D. 2000, ApJ, 539, L9

Fiore, F., et al. 2003, A\&A, 409, 79

Fiore, F., et al. 2008, ApJ, 672, 94

Fruscione, A., et al. 2006, Proc. SPIE, 6270, 6270IV

Garmire, G. P., Bautz, M. W., Ford, P. G., Nousek, J. A., \& Ricker, G. R., Jr. 2003, Proc. SPIE, 4851, 28 
Gebhardt, K., et al. 2000, ApJ, 539, L13

Giacconi, R., et al. 2002, ApJS, 139, 369

Gilli, R., Comastri, A., \& Hasinger, G. 2007, A\&A, 463, 79

Harrison, F. A., Eckart, M. E., Mao, P. H., Helfand, D. J., \& Stern, D. 2003, ApJ, 596,944

Hasinger, G., Miyaji, T., \& Schmidt, M. 2005, A\&A, 441, 417

Hasinger, G., et al. 2007, ApJS, 172, 29

Hornschemeier, A. E., Brandt, W. N., Alexander, D. M., Bauer, F. E., Garmire, G. P., Schneider, D. P., Bautz, M. W., \& Chartas, G. 2002, ApJ, 568, 82

Hornschemeier, A. E., et al. 2003, AJ, 126, 575

Ilbert, O., et al. 2008, ApJ, 690, 1236

Kim, M., et al. 2007, ApJS, 169, 401

Laird, E. S., et al. 2008, ApJS, 180, 102

Lehmer, B. D., et al. 2005, ApJS, 161, 21

Lehmer, B. D., et al. 2006, AJ, 131, 2394

Lilly, S. J., et al. 2007, ApJS, 172, 70

Luo, B., et al. 2008, ApJS, 179, 19

Magorrian, J., et al. 1998, AJ, 115, 2285

Mainieri, V., et al. 2009, A\&A, submitted

Manners, J. C., et al. 2003, MNRAS, 343, 293

Marconi, A., \& Hunt, L. K. 2003, ApJ, 589, L21

McCracken, H. C., et al. 2009, ApJ, submitted

Miyaji, T., et al. 2007, ApJS, 172, 396

Mobasher, B., et al. 2007, ApJS, 172, 117

Moretti, A., Campana, S., Lazzati, D., \& Tagliaferri, G. 2003, ApJ, 588, 696
Murray, S. S., et al. 2005, ApJS, 161, 1

Nandra, K., Mushotzky, R. F., Arnaud, K., Steidel, C. C., Adelberger, K. L., Gardner, J. P., Teplitz, H. I., \& Windhorst, R. A. 2002, ApJ, 576, 625

Nandra, K., et al. 2005, MNRAS, 356, 568

Park, et al. 2006, ApJ, 652, 610

Puccetti, S., et al. 2006, A\&A, 457, 501

Puccetti, S., et al. 2009, ApJS, submitted

Salvato, M., et al. 2009, ApJ, 690, 1250

Sanders, D. B., et al. 2007, ApJS, 172, 86

Schinnerer, E., et al. 2007, ApJS, 172, 46

Scott, K. S., et al. 2008, MNRAS, 385, 2225

Scoville, N., et al. 2007a, ApJS, 172, 1

Scoville, N., et al. 2007b, ApJS, 172, 38

Scoville, N., et al. 2007c, ApJS, 172, 150

Taniguchi, Y., et al. 2007, ApJS, 172, 9

Tremaine, S., et al. 2002, ApJ, 574, 740

Trump, J. R., et al. 2007, ApJS, 172, 383

van Speybroeck, L. P., et al. 1997, Proc. SPIE, 3113, 89

Weisskopf, M. C., Brinkman, B., Canizares, C., Garmire, G., Murray, S., \& Van Speybroeck, L. P. 2002, PASP, 114, 1

Wright, E. L. 2006, PASP, 118, 171

Yang, Y., Mushotzky, R. F., Barger, A. J., \& Cowie, L. L. 2006, ApJ, 645, 68

Yang, Y., Mushotzky, R. F., Steffen, A. T., Barger, A. J., \& Cowie, L. L. 2004, AJ, 128,1501

Zamojski, M. A., et al. 2007, ApJS, 172, 468 